January 12, 2018

\title{
A Lattice Model of Charge-Pattern-Dependent Polyampholyte Phase Separation
}

\author{
Suman DAs, ${ }^{1}$ Adam EISEN, ${ }^{1,2,3}$ Yi-Hsuan LIN, ${ }^{1,4}$ and Hue Sun CHAN ${ }^{1,2, *}$ \\ ${ }^{1}$ Department of Biochemistry, University of Toronto, Toronto, Ontario M5S 1A8, Canada; \\ ${ }^{2}$ Department of Molecular Genetics, University of Toronto, \\ Toronto, Ontario M5S 1A8, Canada; \\ ${ }^{3}$ Department of Mathematics \& Statistics, Queen's University \\ Kingston, Ontario K7L 3N6, Canada; and \\ ${ }^{4}$ Molecular Medicine, Hospital for Sick Children, Toronto, Ontario M5G 0A4, Canada
}

\begin{abstract}
*Corresponding author
E-mail: chan@arrhenius.med.toronto.edu; Tel: (416)978-2697; Fax: (416)978-8548

Mailing address:

Department of Biochemistry, University of Toronto, Medical Sciences Building - 5th Fl., 1 King's College Circle, Toronto, Ontario M5S 1A8, Canada.
\end{abstract}




\begin{abstract}
In view of recent intense experimental and theoretical interests in the biophysics of liquidliquid phase separation (LLPS) of intrinsically disordered proteins (IDPs), heteropolymer models with chain molecules configured as self-avoiding walks on the simple cubic lattice are constructed to study how phase behaviors depend on the sequence of monomers along the chains. To address pertinent general principles, we focus primarily on two fully charged 50-monomer sequences with significantly different charge patterns. Each monomer in our models occupies a single lattice site and all monomers interact via a screened pairwise Coulomb potential. Phase diagrams are obtained by extensive Monte Carlo sampling performed at multiple temperatures on ensembles of 300 chains in boxes of sizes ranging from $52 \times 52 \times 52$ to $246 \times 246 \times 246$ to simulate a large number of different systems with the overall polymer volume fraction $\phi$ in each system varying from 0.001 to 0.1. Phase separation in the model systems is characterized by the emergence of a large cluster connected by inter-monomer nearest-neighbor lattice contacts and by large fluctuations in local polymer density. The simulated critical temperatures, $T_{\mathrm{cr}}$, of phase separation for the two sequences differ significantly, whereby the sequence with a more "blocky" charge pattern exhibits a substantially higher propensity to phase separate. The trend is consistent with our sequence-specific random-phase-approximation (RPA) polymer theory; but the variation of the simulated $T_{\text {cr }}$ with a previously proposed "sequence charge decoration" pattern parameter is milder than that predicted by RPA. Ramifications of our findings for the development of analytical theory and simulation protocols of IDP LLPS are discussed.
\end{abstract}




\section{Introduction}

A central principle of modern biology is that of information ${ }^{1}$. Much of the study of molecular biology aims to ascertain how information embodied in specific sequences of nucleic acids and proteins govern their structures and interactions to serve various physiological functions. Molecular biology of proteins used to focus predominantly on the sequencestructure relationships of globular proteins with highly ordered folded structures. In recent years, however, it has become abundantly clear that intrinsically disordered proteins (IDPs) serve many critical functions, especially those pertinent to cellular signaling and regulation ${ }^{2}$ 4. Unlike globular proteins that fold to an essentially unique structure under physiological conditions, IDPs do not fold by themselves. In the absence of stabilizing interactions with other biomolecules, an IDP can adopt many different structures, i.e., it populates a conformational ensemble. Nonetheless, physics dictates that the conformational distribution in an IDP ensemble is sequence-dependent, not random. Accordingly, to decipher IDPs function biophysically, it is necessary to extend our interest in sequence-structure relationships to a more generalized pursuit of sequence-ensemble relationships.

Some IDPs function not merely via binding interactions that lead to formation of discrete molecular complexes $\frac{56}{6}$. An increasing number of IDPs have now been known to function also at a mesoscopic level by forming droplet-like condensates via liquid-liquid phase separation so as to regulate/stimulate specific set of biochemical reactions. Electrostatic interactions often figure prominently in these phase separation processes (sometimes referred to as coacervation); but other types of interactions, especially cation- $\pi$ and $\pi-\pi$ interactions ${ }^{7}$, can also play significant roles in enabling such functional IDP phase behaviors that, when dysfunctional because, e.g., of mutations of the IDP sequences, can lead to a broad spectrum of diseases 8 . Examples of membraneless organelles - intracellular compartments and subcompartments not bound by lipid membranes - that are underpinned by IDP phase separations include nuage or germ granules ${ }^{15}$, the nucleolus which is the site of ribosome assembly in the nucleus $\frac{16}{}$, and stress granules triggered by heat stress $\frac{17}{}$. Some IDP condensates exhibit liquid-like hydrodynamic properties $\frac{18}{}$, others are observed to be gel-like ${ }^{19}$, or "mature" over time to a state with slower dynamic exchange ${ }^{20}$, including the development of a differentially stabilized core substructure in stress granules ${ }^{21}$ in a process that shares certain resemblance to earlier observations of maturation of coacervated elastin droplets into fibrillar structures ${ }^{22}$. In the case of stress granules, formation of liquid-like droplets can also be a precursor to pathological fibrillization 23 .

IDP phase behaviors are sequence dependent. Gaining physical insights into this sequence dependence is important for progress not only in molecular biology but also in materials science 24 . For an intrinsically disordered region of the DEAD-box RNA helicase Ddx4 - the phase separation of which underlies nuage or germ granules, it has been 
shown experimentally that the wildtype sequence phase separates in vitro and in cells, whereas a charge-scrambled variant of the sequence with the same composition of amino acid residues but a different sequential charge pattern does not $\frac{15}{15}$. In contrast, although the phase behaviors of the Nephrin intracellular domain (NICD) is sequence dependent, the effects of the overall amino acid composition is sufficiently overwhelming that the "precise sequence of NICD appears to matter little"25. To address sequence-dependent IDP phase properties, our group has put forth an analytical formulation ${ }^{26}$ based on the randomphase-approximation (RPA) polymer theory for electrostatic interactions ${ }^{27 / 28}$, affording the first quantitative physical rationalization of the different phase behaviors of Ddx4 and its

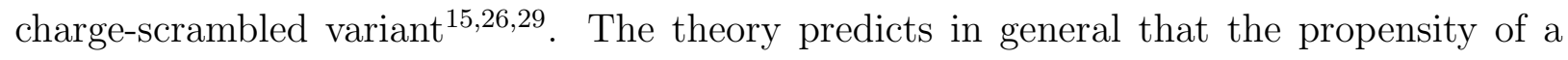
polyampholytic IDP sequence with zero net charge to phase separate is correlated with the "blockiness" and perhaps other yet-unspecified attributes of its charge pattern that are captured by the $\kappa$ (ref. 31) and "sequence charge decoration" (SCD) ${ }^{32} 34$ parameters ${ }^{30}$. The same theory further stipulates that whether the solute populations of two different charged IDP sequences (with zero net charge) present in the same aqueous solution demix upon phase separation is largely governed by the difference in their SCD parameters ${ }^{35}$. Independently, a recent "hybrid" formulation that combines Monte Carlo chain simulations with a Flory-Huggins-like theory tackles how coacervation involving multiple copies of a homopolyanion and a polycation with only half of the monomers charged depends on the sequence of the polycation. Consistent with experiment, the formulation predicts that the tendency to coacervate increases with the blockiness of the polycation ${ }^{36}$. To further substantiate these theoretical/conceptual advances, it is now imperative to assess the approximations that have been invoked to make analytical theories tractable, preferably by direct simulations of explicit-chain models. However, despite notable advances in modeling IDP folding upon binding, using explicit-chain simulation to study IDPs properties in general is still in its infancy $\sqrt{5|6| 37+39}$, especially for phase behaviors which entail sampling a large number of chain molecules. The immense computational cost required dictates that only coarse-grained chain models are currently feasible to be used for phase separation simulations. Insights have been gained, for example, by treating groups of amino acid residues of IDPs as interaction modules ${ }^{16 / 40}$. As a step toward better quantitative understanding, here we develop a simple lattice model to address sequence dependence of IDP phase behavior at the monomer/residue level.

Simple lattice models have made critical contributions to polymer science, beginning at least 70 years ago with the work of Orr in 1947 7 . Early exact enmuerations of chain conformations ${ }^{42}$ have been instrumental in fundamental developments in polymer theory 23 . Lattice models also helped advance studies of micelles (by considering diblock sequences as models for amphiphiles) $)^{44}$, knot theory ${ }^{45}$, RNA conformational statistics $\frac{46[47}{4}$, and DNA topology ${ }^{48}$. As far as proteins are concerned, lattice modeling was pioneered by Gō and 
coworkers. The structure-based approach they introduced in 1975 (ref. 49) and pursued till 1988 (ref. 50) has led to fundamental conceptual advances, including recognizing the importance of local interactions in speeding up folding 5152 , role of nonlocal interactions in folding cooperativity $\$$, and the celebrated "consistency principle" 53154 which is intimately related to the subsequent "principles of minimal frustration" of protein folding 5

Structure-based Gō-like models do not consider the physico-chemical basis of sequence dependence ${ }^{56}$. Physics-based sequence dependence was first introduced into lattice studies of proteins in 1989 by Lau and Dill's 2-letter hydrophobic-polar (HP) model 57 , which is an explicit-chain version of an earlier mean-field HP model of Dill 58 . This construct provides a simple tractable model of the protein sequence-structure mapping ${ }^{59}$ that could readily be explored algorithmically to study protein folding 60 and evolution $\frac{6162}{6}$. At the same time, lattice models were used to address local preference ${ }^{\sqrt[63 \mid 64]{6}}$ and global compactness ${ }^{65 \mid 66}$ of chain conformations as well as the ramifications of their relationships for protein structures 64 . This endeavor led to the first exact enumeration of all compact conformations of a 27 mer configured within a $3 \times 3 \times 3$ cube $^{64}$, a noteworthy model that was subsequently utilized in seminal investigations of the kinetic bottlenecks ${ }^{67}$, the funnel picture ${ }^{68}$, the landscape perspective ${ }^{69}$, and cooperativity ${ }^{70}$ of protein folding as well as the designability/encodability of protein structures ${ }^{71}$. During this period, sequence dependence of protein behaviors was also investigated using 20-letter models on the simple cubic lattice ${ }^{72}$ as well as on face-centered $\operatorname{cubic}^{73}$, diamond ${ }^{74}$ or tetrahedral ${ }^{75}$, and "210" ${ }^{76}$ lattices (see, e.g., refs. 77-79 for reviews). Although the importance of lattice models on the study of folding of small proteins has since diminished - rightfully — as continuum coarse-grained and atomic models that offer higher structural and energetic resolutions become increasingly tractable computationally, simple lattice modeling remains a powerful conceptual tool in the study of protein evolution 80 because of these models' ability to address large-scale sequence-structure relations ${ }^{81}$ in a biophysics-informed manner $\underline{82}$.

Compared to these and many other efforts of using lattice models to study globular proteins, lattice modeling of IDP-like polyampholytes ${ }^{83184}$ had not been extensive. Insofar as phase separations of charged polymers are concerned, grand canonical Monte Carlo simulations have been applied to model phase separations of relatively short charged polymers configured on simple cubic lattices ${ }^{85186}$. The systems considered include fully charged polyelectrolytes of chain lengths $N=3,4,6,8,12,16$, and 24 ( $N=$ number of monomers per chain) with neutralizing counterions in simulation boxes of sizes ranging from $16 \times 16 \times 16$ to $24 \times 24 \times 24$ (ref. 85) as well as fully charged polyampholytes with zero net charge of chain lengths $N=2, N=4$ (a diblock sequence), $N=8$ (four different sequences), and $N=16$ (three different sequences) configured in boxes of sizes ranging from $14 \times 14 \times 14$ to $24 \times 24 \times 24$ (ref. 86). Because these chain lengths are much shorter than those of IDPs involved in functional phase separations, in order to better connect 
lattice modeling to experimental IDP phase behavior, it would be desirable to conduct similar studies for polyampholytes of longer chain lengths. However, unlike homopolymer lattice systems with only nearest-neighbor interactions ${ }^{87}$, applying the grand canonical Monte Carlo method to larger N's is technically problematic for polyampholytes, because of a sharply increasing rejection rate for attempted chain transfers with increasing $N$ (ref. 85, 86). Here we take an alternate "brute-force" approach. As a case study, we apply direction simulations two fully charged $N=50$ sequences with zero net charge but significantly different charge patterns ${ }^{30131}$ to verify that the sequences with a more blocky charge pattern indeed phase separates much more readily than the strictly alternating sequence with minimum blockiness. The differential effect we observe is significant; but it is also noteworthy that the phase separation tendency seen in our direct explicit-chain simulations is less sensitive to charge pattern ${ }^{31} 33$ than that stipulated by RPA theory 29130 , underscoring that quantitative predictions of the theory need to be treated with caution.

\section{Models and Methods}

Model polymer chains are configured as self-avoiding walks on a simple cubic lattice (coordination number 6). Each polymeric bond connects two monomers that are nearest neighbors on the lattice. In other words, the length of the bond in our model is fixed as in most lattice protein models ${ }^{646768}$. Unlike the bond fluctuation mode ${ }^{88}$ used in ref. 85, our model only allows bonds in the $(0,0,1)$ direction and its five rotations and inversions but does not allow bonds in the $(0,1,1)$ and $(1,1,1)$ and their rotations and inversions. The present simulations of the configurations of multiple polymer chains are conducted in cubic boxes with periodic boundary conditions.

For any two different monomers labeled $\mu, i$ and $\nu, j(\mu, \nu=1,2, \ldots, n$ label the polymer chains where $n$ is the total number of chains in the simulation system, $i, j=1,2, \ldots, N$ label the $N$ monomers along each chain) with charges $\sigma_{\mu i}, \sigma_{\nu j}$, their electrostatic interaction is given by $\left(U_{\mathrm{el}}\right)_{\mu i, \nu j}=\left(l_{B} \sigma_{\mu i} \sigma_{\nu j} / r_{\mu i, \nu j}\right) \exp \left(-r_{\mu i, \nu j} / r_{\mathrm{S}}\right)$, where $r_{\mu i, \nu j}$ is the spatial distance between the two monomers, $l_{B}=e^{2} /\left(4 \pi \epsilon_{0} \epsilon_{\mathrm{r}} k_{\mathrm{B}} T\right)$ is Bjerrum length, $e$ is elementary electronic charge, $\epsilon_{0}$ is vacuum permittivity, $\epsilon_{\mathrm{r}}$ is relative permittivity (dielectric constant), $k_{\mathrm{B}}$ is Boltzmann constant, and $T$ is absolute temperature. The total potential energy is thus given by $\sum_{\mu=1}^{n} \sum_{\nu=1}^{n} \sum_{i=1}^{N} \sum_{j=1}^{N}\left(1-\delta_{\mu \nu} \delta_{i j}\right)\left(U_{\mathrm{el}}\right)_{\mu i, \nu j}$, where the Kronecker symbol $\delta_{i j}=1$ for $i=j, \delta_{i j}=0$ for $i \neq j$ and the first factor in the summation serves to exclude self-interaction terms. Here we use $\epsilon_{\mathrm{r}}=80$, which corresponds to that of the water solvent, and a lattice constant $a=3.1 \AA$ such that $a^{3}$ is approximately the size of a water molecule; and $r_{\mathrm{S}}$ is the screening length in the model. For computational efficiency, we impose a $3 a$ cutoff for the interaction, i.e., $\left(U_{\mathrm{el}}\right)_{\mu i, \nu j}=0$ for $r_{\mu i, \nu j}>3 a$, and employ a temperature-independent $r_{\mathrm{S}}=10 \AA$. The model polymer bond length is equal to $a$ in this construct. Results for 
(a)

ЕRеRERERERERERERERERERERERERERERERERERERERERERERER
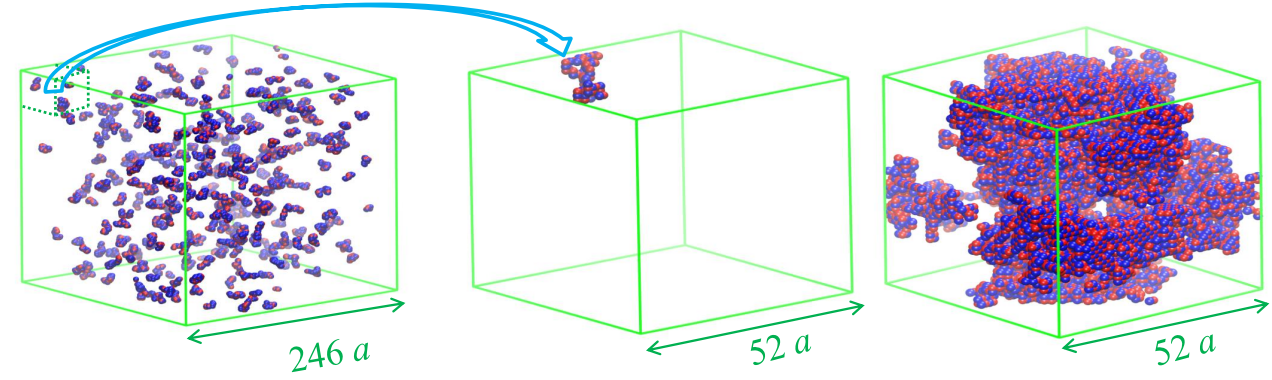

(b)

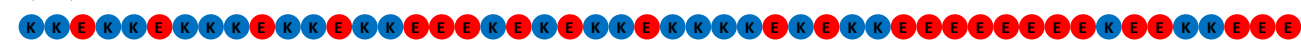
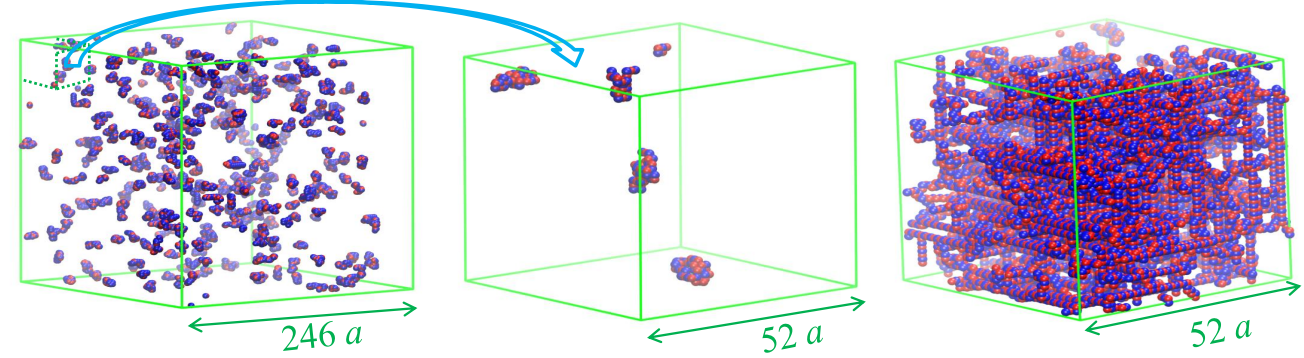

FIG. 1: Examples of systems simulated in this work for sequences sv1 (a) and sv15 (b). Positively $(\mathrm{K})$ and negatively (E) charged residues (monomers) are depicted, respectively, as blue and red beads. Snapshots of simulation boxes are taken at (a) $T=200 \mathrm{~K}$ and (b) $800 \mathrm{~K}$. The snapshots are representative of chain configurations below (left) and above (right) the respective system's critical concentration $\phi_{\mathrm{cr}}$. Each of these four boxes contains 300 polymer chains. The simulation boxes are of sizes $246 a \times 246 a \times 246 a$ (all box sizes are provided in units of $a^{3}$ hereafter) (left, overall polymer volume fraction $=0.001$ ) and $52 \times 52 \times 52$ (right, overall polymer volume fraction $=0.1$. The middle drawings are zoom-in images of a part of the simulation boxes on the left (as indicated by the arrows) to facilitate comparison with the snapshots on the right by showing them in the same length scale. Note that the present color code for the $+/-$ monomers of 50mer $\mathrm{KE}$ sequences (red for -; blue for + ) is identical to that in refs. 30 and 35 . The statements in the captions for Figure 1 of ref. 30 and Figures 2 and 3 of ref. 35 that red is for + and blue is for monomers were typographical errors that have no bearing on the results in refs. 30 and 35 .

other polymer bond lengths (with a rescaled $r_{\mathrm{S}}$ ) may be obtained from the present data by rescaling the temperature. All simulated results reported here are for ensembles of $n=300$ identical polymer chains configured in simulation boxes of various sizes.

The bulk of the present simulation effort is focused on two fully charged polyampholytes with zero net charge $\left(\sigma_{i}, \sigma_{j}= \pm 1, \sum_{i=1}^{N} \sigma_{i}=0\right.$, wherein the $\mu, \nu$ indices can be dropped from the $\sigma$ 's because our simulated systems are restricted to ensembles of identical sequences). The sequences correspond to those labeled as sv1 and sv15 among the thirty KE sequences first considered in ref. 31 (Fig. 1). Here K and E stand for lysine and glutamic acid, respec- 
tively (note that $\mathrm{K}$ also denotes degree Kelvin in contexts that should entail no confusion). The charge patterns of these two sequences are significantly different, as reflected by their $\kappa$ parameters $(\kappa=0.0009 \text { for sv1 and } \kappa=0.1354 \text { for sv15 })^{31}$ as well as their sequence charge decoration $(\mathrm{SCD})$ parameters ${ }^{32}$, where

$$
\mathrm{SCD} \equiv \frac{1}{N} \sum_{i=1}^{N} \sum_{j=i+1}^{N} \sigma_{i} \sigma_{j} \sqrt{j-i}
$$

with $\mathrm{SCD}=-0.41$ for $\mathrm{sv} 1$ and $\mathrm{SCD}=-4.35$ for sv15 $5^{32 \mid 35}$. In view of the intensive computation required to simulate the phase behaviors of these sequences, investigation of other $N=50$ sequences with different KE patterns is left to future efforts.

Monte Carlo simulations are used to sample chain configurations 89 . The initial configuration of each of our simulation systems is prepared by randomly placing all the polymers as fully extended chains along the three Cartesian axes of the simulation box in equal numbers. The system is first equilibrated for $10^{7}$ simulation steps, to be followed by a production run with duration ranging from $2 \times 10^{7}$ to $10^{8}$ simulation steps. Each simulation step is an attempted move performed on a randomly chosen polymer chain and at a randomly chosen location along the chain. Move acceptance is based on the Metropolis criterion. Excluded volume is enforced by disallowing any two chain monomers to occupy the same lattice site, i.e., attempted moves that would result in such disallowed configurations are rejected. The attempted move is chosen stochastically among four types of moves with the following percentage statistical weights: diagonal (kink jump, 40\%), crankshaft (40\%), pivot (including end rotation, 10\%), and reptation (10\%). The first two types of moves are local motions that only involve a small number of monomers, whereas the latter two types of moves entail global motions that can potentially relocate a large number of monomers (up to order $N)$. The acceptance rates of these moves at the lowest simulation temperature $(200 \mathrm{~K})$ are approximately $12 \%, 2 \%, 4 \%$, and $0.25 \%$, respectively; the rates are higher at higher temperatures. The function gsl_rng.h and the random number generator mt19937 are used for the simulations.

We perform two sets of extensive simulations for both sequences sv1 and sv15. The first set of simulations is geared toward addressing the low-concentration side of the coexistence phase boundary by observing whether a percolating polymer cluster develops in the simulation box (see below). These simulations are performed at 9 temperatures (200 $\mathrm{K}, 300 \mathrm{~K}, \ldots, 1000 \mathrm{~K}$ ) and 19 different overall polymer volume fractions (defined as the number of monomers divided by the total number of lattice sites in the simulation box), namely $0.001,0.002, \ldots, 0.009$, and $0.01,0.02, \ldots, 0.09,0.1$ by varying the simulation box size from $52 \times 52 \times 52$ to $246 \times 246 \times 246$. Illustrative snapshots of the simulated polymer configurations are provided in Fig. 1. The second set of simulations is designed for determining the full coexistence phase boundary by observing spatial variations and 


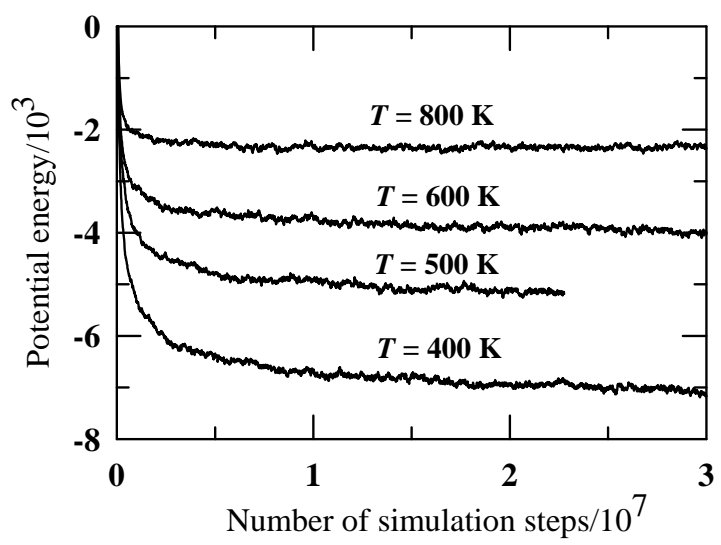

FIG. 2: Evolution and gradual stabilization of potential energy (in units of $k_{\mathrm{B}} T$ ) as a function of number of simulation steps. Shown results are for 300 copies of sequence sv15 in a simulation box of size $52 \times 52 \times 52$ at four different temperatures.

overall distribution of polymer density (see below). These simulations are performed at a fixed overall polymer volume fraction of 0.1 (by using only $52 \times 52 \times 52$ simulation boxes) for 17 different temperatures $(200 \mathrm{~K}, 250 \mathrm{~K}, 300 \mathrm{~K}, 350 \mathrm{~K}, \ldots, 1000 \mathrm{~K})$. We monitor the evolution of total potential energy as each simulation proceeds to ensure, to the extent possible within our computational resources, that the system reaches a quasi-steady state during the production stage of our simulation by observing a near-leveling of the total potential energy. Nonetheless, at relatively lower temperatures, it is evident that the system is evolving very slowly — with the potential energy stabilizing very gradually - even after a large number of simulation steps (see examples in Fig. 2). The ramification of this behavior will be addressed below.

\section{Results and Discussion}

\section{Temperature- and Concentration-Dependent Distributions of Polymer} Density. We begin by examining our first set of simulations. The temperatures and the overall polymer volume fractions ( $\phi$ values, referred to interchangeably as concentrations below) of a subset of the simulated systems are represented by the grid points marked by circles or squares in Fig. 3. For each system, we determine whether there exists a percolating cluster connected by intrachain connectivity and interchain nearest-neighbor contacts that encompasses $\geq 80 \%$ of the polymer chains. Snapshots of systems with and without such a cluster are provided by the examples on the right and left, respectively, of Fig. 1. We arrived at the choice of using $\geq 80 \%$ as an intuitive, putative criterion for phase separation after monitoring a variety of clusters under different simulation conditions, and expect 


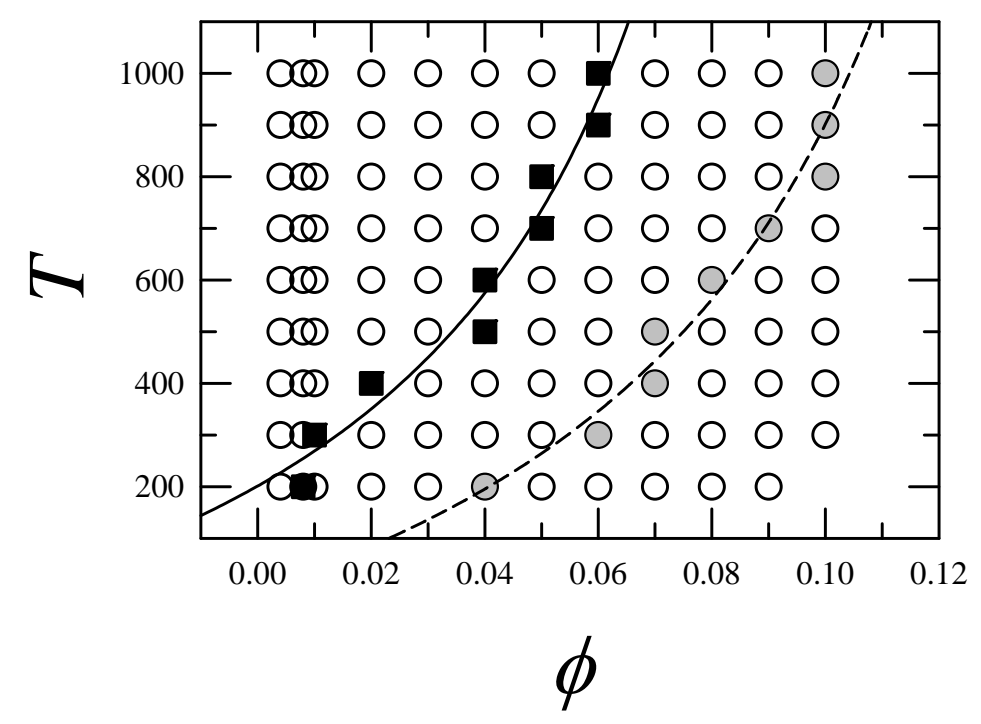

FIG. 3: Estimating the dilute side of the phase boundary by the presence or absence of a large polymer cluster. Each circle or square represents a $(T, \phi)$-system we simulated for sequences sv1 and sv15 (separately) to ascertain whether a cluster consisting of $\geq 80 \%$ of the polymer chains is present in the simulation box. Such a cluster exists for sequences sv1 and sv15 in all $(T, \phi)$ systems marked, respectively, by the filled gray circles and filled black squares as well as systems with larger $\phi$ values plotted to their right. Fitted continuous curves (solid and dashed curves for filled black squares and gray circles respectively) are guides for the eye. $T$ is in units of $\mathrm{K}$.

that reasonable variations of this criterion would produce similar results. The investigative protocol here is logically akin to the experiments that rely on observation/no observation of droplet formation by microscopy, an experimental technique that is commonly utilized for ascertaining conditions for IDP phase separation (see, e.g., Figure 3B of ref. 23). The result of our extensive exploratory study is shown in Fig. 3. It shows a clear sequence effect. At every temperature we simulated, the sequence with a more blocky charge pattern (sv15, squares in Fig. 3) begets such a cluster at a lower concentration $\phi$ than the strictly alternating (non-blocky) sequence sv1 (gray circles in Fig. 3). It is quite remarkable that at sufficiently low temperature $(200 \mathrm{~K})$, a very low $\phi<0.01$ is sufficient to induce formation of a cluster for sequence sv15 that encompasses $\geq 80 \%$ of the chains.

In addition to monitoring the formation of a large polymer cluster, phase behavior in the simulation systems is addressed by characterizing fluctuations in polymer density. For each system in the second set of simulations, we determine, as a function of position $(x, y, z)$ within the (large) simulation box, the number of sites within small cubic volumes (small boxes) that are occupied by the polymer chains (Fig. 4). The ratio of this number with the small cubic volume is the local polymer density (or, equivalently, local polymer con- 
centration or local polymer volume fraction), denoted as $\phi(x, y, z)$ hereafter. Because of periodic boundary conditions, the total number of small boxes we used to sample local density is equal to the number of lattice sites in the simulation box. Most of the results on local polymer density presented below are obtained by using small boxes of size $8 \times 8 \times 8$. Corresponding results from using small boxes of sizes $3 \times 3 \times 3, \ldots, 7 \times 7 \times 7$ are similar. At high temperatures, local polymer density is quite narrowly distributed around a single sharp peak (see Fig. 5a for an example, note that the vertical scale is logarithmic). In contrast, at low temperatures, distributions of local polymer density typically consist of one peak at very low density and a broad plateau-like regime with a very gradual decreasing trend that extends to very high density (Fig. 5b), a feature indicative of significant heterogeneity in concentration within the simulated ensemble. Fig. 5 also shows that these trends are largely insensitive to the size of small boxes within the range from $3 \times 3 \times 3$ to $8 \times 8 \times 8$ we have considered. Qualitatively, the resulting distributions exhibit very similar shapes. Quantitatively, the lowering of the peak in Fig. 5a and the plateau region in Fig. 5b (by approximately one order of magnitude) when the size of the small box is increased from $3 \times 3 \times 3$ to $8 \times 8 \times 8$ is attributable mainly to two factors: (i) The increase in the number of possible polymer occupancies from $3^{3}=27$ to $8^{3}=512$ (whereas the total number of small boxes is fixed) means that the support of the distribution is stretched by a factor of $\approx 20$. By itself, this consideration would argue that, relative to the distributions for the case with small box size $3 \times 3 \times 3$ in Fig. 5 , a multiplicative factor of $\approx 20$ should be applied to the distributions plotted in Fig. 5 for the case with small box size $8 \times 8 \times 8$ to compare the distributions on the same footing, i.e., to bring them to the same normalization. (ii) In the hypothetical limit of the size of the small boxes approaching that of the simulation box itself, the distributions would become uniform. This consideration implies that, as the size of the small box increases, a general "flattening" tendency of the distribution of polymer occupany is expected. Taking the results in Fig. 5 (peak/plateau for the $3 \times 3 \times 3$ case $\approx 10$ times higher than those for the $8 \times 8 \times 8$ case) and consideration (i) above - i.e., a factor of $\approx 20$ should be multiplied to the distributions for the $8 \times 8 \times 8$ case to bring them to the same normalization conditions as those for the $3 \times 3 \times 3$ case - together, this expected flattening effect apparently leads to a reduction of the peak and plateau heights of the distributions for small box size of $8 \times 8 \times 8$ from those for small box size of $3 \times 3 \times 3$ by a factor of $\approx 2$ if they are compared on a normalized footing.

For a given simulation system with a given size of the small box for sampling local polymer density, we define $\phi(x)$ as $\sum_{y, z} \phi(x, y, z) / L^{2}$, where $L$ is the linear dimension of the cubic simulation box. Thus $\phi(x)$ is an average local polymer density, or equivalently the average polymer density over a slab on the $y-z$ plane with a thickness equal to the linear dimension of the sampling small box; $\phi(y)$ and $\phi(z)$ are defined analogously (Fig. 6a). An overall average distribution $\langle\phi\rangle_{u}$ for monitoring the spatial variation of polymer density is 


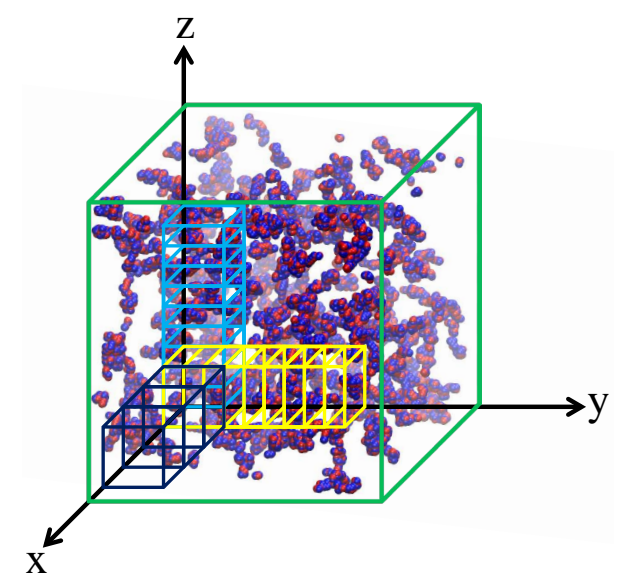

FIG. 4: Sampling local polymer density. As described in the text, small boxes are placed at all positions within the simulation (large) box to determine local volume fraction of the model polymer chains. The snapshot shows 300 copies of sequence sv1 in a $115 \times 115 \times 115$ simulation box at $T=1000 \mathrm{~K}$. The small boxes shown are of size $8 \times 8 \times 8$.

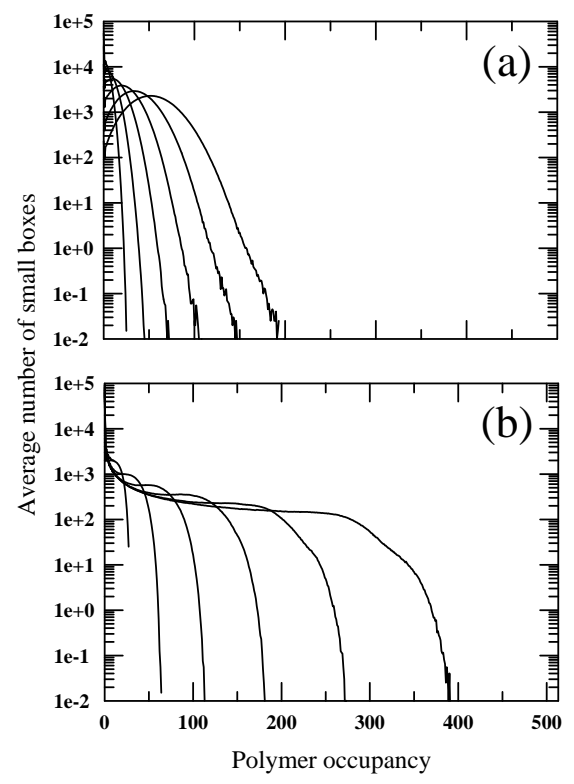

FIG. 5: Distributions of local polymer density. Examples are shown for 300 copies of sequence sv1 simulated (a) above $T_{\text {cr }}\left(\right.$ at $600 \mathrm{~K}$ ) and (b) below $T_{\text {cr }}$ (at $200 \mathrm{~K}$ ) in a $52 \times 52 \times 52$ simulation box. The curves in both (a) and (b) are for small boxes of sizes (from left to right) $3 \times 3 \times 3,4 \times 4 \times 4$, $5 \times 5 \times 5,6 \times 6 \times 6,7 \times 7 \times 7$, and $8 \times 8 \times 8$. Polymer occupancy (horizontal variable) is the number of lattice sites, within a given small box, that are occupied by monomers of the simulated chains. Polymer volume fraction is equal to polymer occupancy divided by the size of the small box. Each average number of small boxes with a given polymer occupancy is computed from 199 snapshots. 


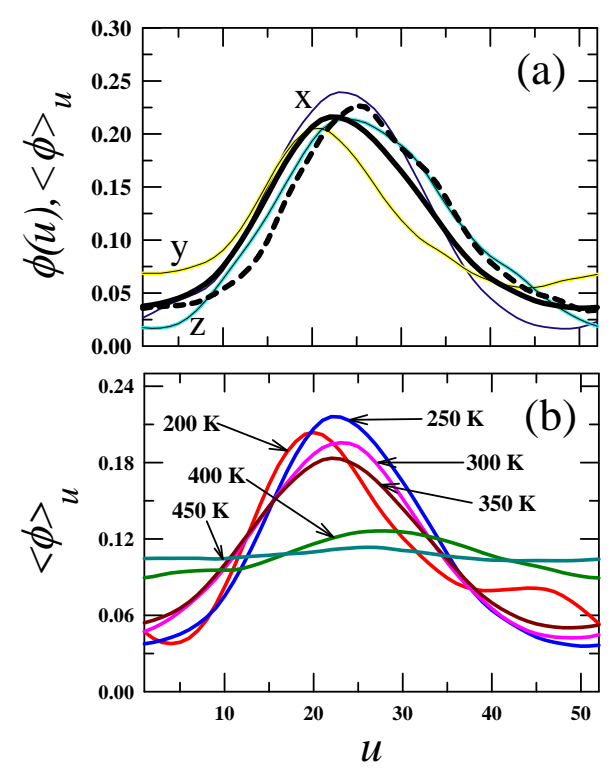

FIG. 6: Variation in local polymer density. Results are for 300 copies of sequence sv1 in a $52 \times 52 \times 52$ simulation box. Except specified otherwise, local polymer density is determined using small boxes of size $8 \times 8 \times 8$. The horizontal variable $u$ stands for $x, y$ or $z$ (as in Fig. 4). (a) Vertical variable $\phi(u)$ is the average volume fraction as a function of $x, y$ or $z$ at $T=250 \mathrm{~K}$ (plotted by the thin colored curves as marked); $\langle\phi\rangle_{u} \equiv[\phi(x)+\phi(y)+\phi(z)] / 3$ is their average (thick solid black curves). The corresponding $\langle\phi\rangle_{u}$ computed by using small boxes of size $3 \times 3 \times 3$ (thick dashed black curve) is also plotted for comparison. (b) Average $\langle\phi\rangle_{u}$ of the same system computed using $8 \times 8 \times 8$ small boxes at different simulation temperatures.

defined as $[\phi(x)+\phi(y)+\phi(z)] / 3$, where $x=y=z=u$ (Fig. 6). The example in Fig. 6a illustrates that, for the systems considered in our second set of simulations, the highest and lowest $\langle\phi\rangle_{u}$ values are not much affected by the size of small boxes for sampling local polymer density (the thick solid and dashed curves in Fig. 6a have very similar shapes). The average distribution $\langle\phi\rangle_{u}$ is bell-shaped at low temperatures (Fig. 6a), which is indicative of the presence of a cluster with locally elevated polymer density. Not unexpectedly, $\langle\phi\rangle_{u}$ is essentially flat at high temperatures when the polymers are spatially more evenly distributed (Fig. 6b). The trend is essentially identical for an alternate order parameter $z_{c}$ for local density (Fig. 7). Defined as the number of nearest-neighbor contacts per monomer ("contacts" between sequential neighbors $\mu, i$ and $\mu, i+1$ along a chain not counted), $z_{c}$ is analogous to the coordination number collective variable defined in ref. 90.

Effects of Charge Pattern and Net Charge on Phase Separation. We now proceed to construct phase diagrams from information gleaned from the local polymer density simulations. Because the overall volume fraction $\phi=0.1$ in these simulations is sufficiently 


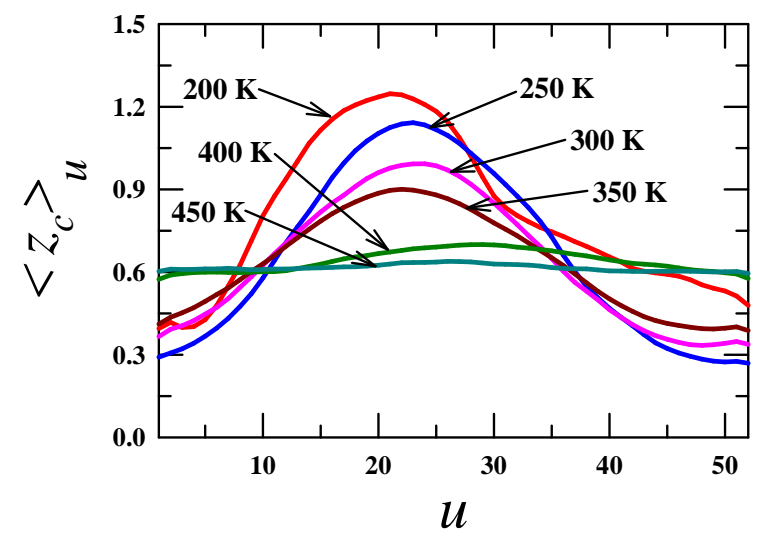

FIG. 7: Spatial variation of polymer density monitored by the average number of monomermonomer contacts. For chains configured on the simple cubic lattice, $0 \leq z_{c} \leq 4$ for the monomers not at chain ends and $0 \leq z_{c} \leq 5$ for the monomers at chain ends. The average $\left\langle z_{c}\right\rangle_{u} \equiv\left[\left\langle z_{c}\right\rangle_{x}+\right.$ $\left.\left\langle z_{c}\right\rangle_{y}+\left\langle z_{c}\right\rangle_{z}\right] / 3$ where $\left\langle z_{c}\right\rangle_{x}$ is the average over the monomers on the $y-z$ plane at $x$, etc. Shown here are $\left\langle z_{c}\right\rangle_{u}$ computed at different temperatures for the system in Fig. 6 .

high, polymer clusters always span the entire length of each of the three dimensions of the simulation box (see, e.g., snapshots on the right in Fig. 1). This feature allows us to estimate the coexisting volume fractions for systems that are clearly phase separated (e.g., those at or below $350 \mathrm{~K}$ in Fig. $6 \mathrm{~b}$ and Fig. 7) by identifying the condensed-phase volume fraction as $\max \left[\langle\phi\rangle_{u}\right]$ and the dilute-phase volume fraction as $\min \left[\langle\phi\rangle_{u}\right]$. This procedure leads to the phase diagrams for sequences sv1 and sv15 in Fig. 8, wherein data points are plotted for temperatures with $\max \left[\langle\phi\rangle_{u}\right]-\min \left[\langle\phi\rangle_{u}\right]>0.01$. Although the accuracy of each individual phase diagram is limited by the finite sizes of the simulation systems and numerical uncertainties caused by extreme slow equilibration at low temperatures (see below), the results in Fig. 8 are adequate for comparing the significantly different phase behaviors of the two sequences. Qualitatively consistent with expectation and theory, sequence sv15 has a significantly higher tendency to phase separate than sv1. The critical temperature, $T_{\mathrm{cr}}$, of sv15 is estimated to be approximately 1.9 times that of sv1. Quantitative comparison of our simulation results with predictions from analytical theories is provided below under the next subheading. It is instructive to note the differences between the phase boundaries estimated by local polymer density (data points and thick curves in Fig. 8) and the putative phase boundaries suggested by observation of a percolating cluster (Fig. 3 and thin curves in Fig. 8), with the latter extending to temperatures above the estimated $T_{\mathrm{cr}}$ 's of the former. This finding implies that the existence of a percolating cluster is not sufficient, in general, for a clearly bimodal distribution of local polymer density. In other words, loosely connected polymer clusters can exist above $T_{\mathrm{cr}}^{*}$. For $T<T_{\mathrm{cr}}$, the difference between the two types 


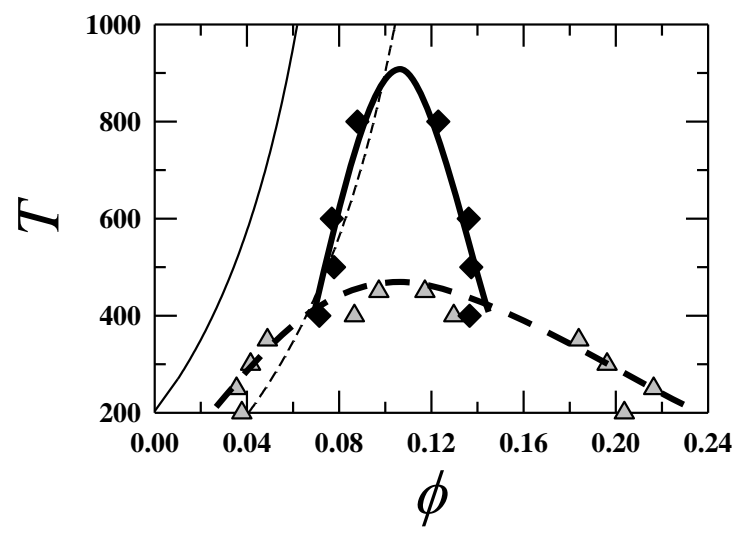

FIG. 8: Simulated polyampholyte phase diagrams exhibit significant dependence on charge pattern. Data points depicted by symbols are obtained from local polymer density analysis for sequences sv1 (gray triangles) and sv15 (black diamonds) using 300 polymer chains in a $52 \times 52 \times 52$ simulation box. The thick dashed and solid curves are empirical fits, respectively, for the plotted sv1 and sv15 data points. Critical temperatures $\left(T_{\mathrm{cr}}\right)$ are estimated by the peak temperatures of the fitted curves. The thin dashed and solid curves are putative phase boundaries obtained in Fig. 3 for sv1 and sv15 by the existence of a $\geq 80 \%$ percolating polymer cluster. $T$ is in units of K.

of estimated phase boundaries may be partly attributed to the fact that phase boundaries are not infinitely sharp ${ }^{9192}$, and that the width of the boundary region is expected to be pronounced for finite-size explicit-chain model simulation systems.

As mentioned above, equilibration in our simulation systems for sv15 is extremely slow for $T \leq 400 \mathrm{~K}$ (Fig. 2): whereas potential energy essentially levels off toward the end of the simulation (at $3 \times 10^{7}$ steps) for $T=800 \mathrm{~K}$, it is still decreasing for $T=400 \mathrm{~K}$, albeit very gradually with a slope $\approx-1.6 \times 10^{-5}$. This situation could be a mere consequence of a basic limitation of lattice chain models. For instance, lattice protein chain models of the HP variety with attractive hydrophobic-like interactions are prone to be trapped kinetically ${ }^{93}$, leading to glassy dynamics and making it difficult to access their lowest-energy states via common Monte Carlo chain moves 94 . Even Gō models with structurally highly specific interactions encounter transient kinetic traps 95 . Nonetheless, inasmuch as Monte Carlo chain moves mimick physical Brownian motions ${ }^{93196}$, the slow dynamics suggested by some of our model systems can be reflective of physical behaviors of real polyampholytes. Some IDP condensates require energy input via ATP-dependent processes (not considered in our model) to maintain an "active" liquid-like state ${ }^{8 \mid 13}$. Some IDP condensates are known to undergo functional maturation ${ }^{20 \mid 22}$ or pathological fibrillization ${ }^{23}$ to condensed states with slower dynamics. If our model is seen as capturing some of the latter slow-dynamics behaviors in a rudimentary manner, the sequence-dependent phase diagrams in Fig. 8 would be 
relevant to experimental phase behaviors determined at a time scale comparable to that for the onset of maturation, notwithstanding the observation that some of our low-temperature model systems have not fully equilibrated. An obvious feature of lattice polymer models is their imposition of a spatial order that may otherwise be absent. For lattice models of globular proteins, it has been argued that this spatial order can play a structural role similar to the hydrogen bonding network in the hydrophobic cores of folded structures 97.99 . Disorder-to-order transitions to solid-like phases have been reported in previous simulations of lattice polyampholytes ${ }^{86}$. Whether such features of the model can be used to gain insights into IDP maturation and fribillization deserves further study.

The main goal of our effort here is to explore general principles of sequence-dependent phase behaviors of polyampholytes and other heteropolymers and to use our simulation results to assess analytical theories (see below). Quantitative comparisons with experiment is not our aim. Nonetheless, we should comment upon our consideration of a temperature range far exceeding - even just nominally - that of liquid aqueous solutions under atmospheric pressure (see, e.g., the $T=200 \mathrm{~K}$ to $1000 \mathrm{~K}$ range in Fig. 8). The high simulated $T_{\text {cr }}$ values (in $\mathrm{K}$ ) are a consequence of the strong electrostatic interactions entailed by the two fully charged polyampholytes. Nonetheless, the same trend of sequence dependence is expected to hold for a pair of sequences with similar charge patterns but lower charge densities when, e.g., the charged monomers are interspersed among neutral monomers along the chain. Our results are relevant to those situations. For instance, because the interaction strength scales as much as the square of charge density (or a somewhat lower exponent depending on the sequence $\left.{ }^{29}\right)$, a polyampholyte with a similar chain length and similar charge pattern of sv15 but with, e.g., $\approx 1 / \sqrt{3}$ of sv15's charge density could reduce $T_{\text {cr }}$ to $\approx 1 / 3$ that for sv15 (i.e., from $\approx 900 \mathrm{~K}$ to $\approx 300 \mathrm{~K}$ ). As discussed above, a scaling down of temperature can also ensue if we apply the current models to polyampholytes with monomer-monomer bond lengths $>3.1 \AA$. A similar interpretative perspective applies to the study of model hydrophobic sequences below.

As another example of sequence-dependent phase behaviors, we compare the simulated phase diagrams of two $N=50$ sequences, termed $\mathrm{h} \phi$ and $\mathrm{h} \phi-$, that are constructed for modeling, respectively, an all-hydrophobic sequence and a predominantly hydrophobic sequence with four embedded negatively charged monomers (Fig. 9, top drawings). Hydrophobic interaction with short spatial range is modeled by a nearest-neighbor attractive contact energy between any pair of hydrophobic monomers on the same chain or on different chains but not sequentially adjacent along a chain. The magnitude of this contact energy is equal to $1 / 3$ of the magnitude of pairwise electrostatic energy in our polyampholyte model at nearestneighbor spatial separation. Unlike charged monomers, hydrophobic monomers have no interaction (energy $=0$ ) beyond nearest neighbor in our model. Hydrophobic interactions are effective, solvent-mediated and thus they are temperature-dependent $\frac{100[101}{\text {. However, }}$ 


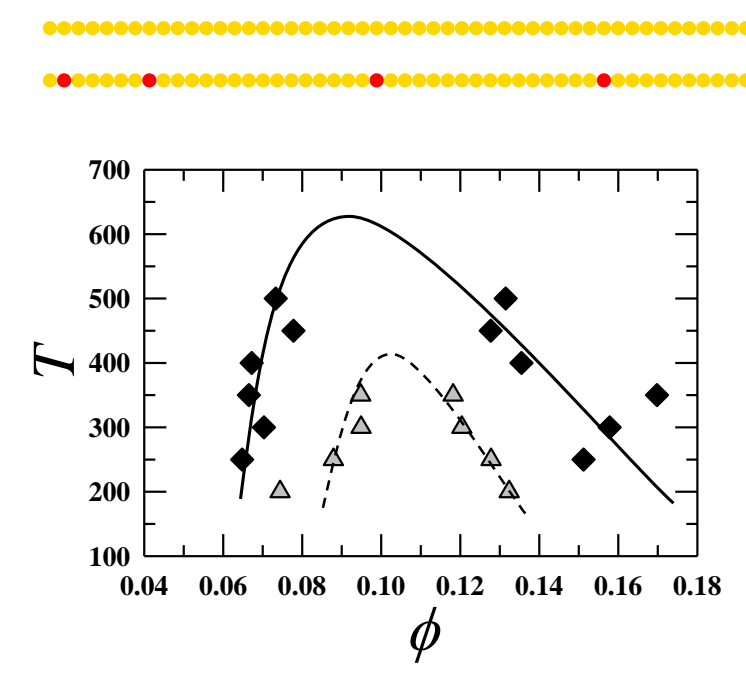

FIG. 9: Simulated phase diagrams for the $\mathrm{h} \phi$ and $\mathrm{h} \phi$ - sequences. The model sequences are shown at the top, wherein hydrophobic and negatively charged monomers are depicted, respectively, in golden and red. $\mathrm{h} \phi$ is all hydrophobic; $\mathrm{h} \phi$ - contains four negative charges. Data points depicted by symbols in the plot are obtained from local polymer density analysis for h $\phi$ (black diamonds) and $\mathrm{h} \phi-$ (gray triangles) using 300 polymer chains in a $52 \times 52 \times 52$ simulation box. The solid and dashed curves are empirical fits, respectively, for the plotted $h \phi$ and $h \phi-$ data points. $T$ is in units of $\mathrm{K}$.

because our main interest here is the effect of sparsely distributed charges on phase behaviors by comparing two sequences with the same hydrophobic background, not the effect of hydrophobicity itself, we use a temperature-independent model for hydrophobic interactions for simplicity. The interaction among the charged monomers in sequence $\mathrm{h} \phi-$ follows that of the above polyampholyte model. We conduct simulation at $250 \mathrm{~K}, 300 \mathrm{~K}, \ldots, 500 \mathrm{~K}$ for sequence $\mathrm{h} \phi$ and $200 \mathrm{~K}, 250 \mathrm{~K}, 300 \mathrm{~K}$, and $350 \mathrm{~K}$ for sequence $\mathrm{h} \phi-$ and apply the local polymer density method described above using small boxes of size $8 \times 8 \times 8$. The phase diagrams we estimated from these simulations are provided in Fig. 9.

Not surprisingly, the $\mathrm{h} \phi$ - sequence with embedded negative charges (gray triangles in Fig. 9) has a significantly lower propensity to phase separate (i.e., it has a lower $T_{\text {cr }}$ ) than the all-hydrophobic $\mathrm{h} \phi$ sequence (black diamonds in Fig. 9) because of the repulsive electrostatic interactions among the embedded negative charges in $\mathrm{h} \phi-$. This example is extremely simple, yet it illustrates how phosphorylations can be used to regulate IDP phase separation in the living cell. Phosphorylations add negative charges to an IDP and thus can modulate its conformational dimensions ${ }^{34}$ and its "polyelectrostatic" interactions with other biomolecules ${ }^{1021103}$. Experiments on the 163-residue N-terminal low-complexity domain of the RNA-binding protein Fused in Sarcoma (FUS LC) ${ }^{104}$ show that phosphorylations of this IDP disrupt its phase separation. A phosphomimetic variant 


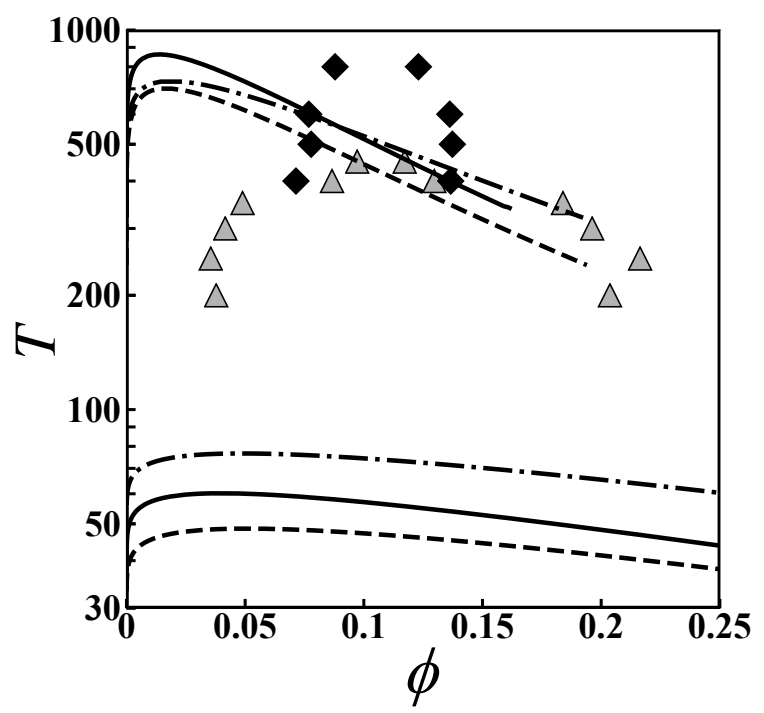

FIG. 10: Comparing simulation results with RPA theories. Data points for the simulated phase boundaries of sequences sv1 (gray triangles) and sv15 (black diamonds) from Fig. 8 are compared with coexistence phase boundaries predicted by three related RPA theories: salt-free RPA (solid curves), RPA with monovalent salt ions, where salt volume fraction $\phi_{s}=0.0034$, i.e., $[\mathrm{NaCl}] \approx 200$ $\mathrm{mM}$ (dashed curves), and RPA for Coulomb potential with a temperature-independent screening (dashed-dotted curves). In every case, the theory-predicted phase boundary for sv15 is above (has a higher critical temperature $T_{\text {cr }}$ than) that for sv1. All RPA-predicted phase boundaries shown here are determined by using a monomer length scale $a=3.1 \AA$ and relative permittivity $\epsilon_{\mathrm{r}}=80$ (ref. 29). $T$, in units of $\mathrm{K}$, is provided by a logarithmic scale to facilitate comparison of ratios of $T_{\text {cr }}$ 's for the two sequences.

of FUS LC, with twelve glutamic acid (E) substitutions for serine or threonine at positions $7,11,19,26,30,42,61,68,84,87,117$, and 131 (the variant is termed FUS LC 12E) also disrupts phase separation ${ }^{105}$. With this in mind, our $\mathrm{h} \phi$ and $\mathrm{h} \phi-$ may be viewed as toy models, respectively, of FUS LC and FUS LC 12E (the spacings of the four negative charges at positions $2,8,24$, and 40 of our $\mathrm{h} \phi$ - are chosen to mimick the distribution of $\mathrm{E}$ residues along FUS LC 12E) for demonstrating the basic principles of how phosphorylations can functionally modulate IDP phase behaviors.

\section{Comparisons with Analytical Theories and Correlations with Charge Pattern}

Parameters. We now compare our explicit-chain simulated phase diagrams for sequences sv1 and sv15 against RPA predictions for the two polyampholyte sequences (Fig. 10). We consider three different sequence-dependent RPA formulations: (i) Salt-free RPA, which corresponds to the $\rho_{s}=\rho_{c}=0$ case in ref. 29, where $\rho_{s}$ and $\rho_{c}$ are the average number densities of salt and counterions, respectively. (ii) RPA with monovalent salt ions, with 
volume fraction of positive or negative salt ion, $\phi_{s}=\rho_{s} a^{3}=0.0034$. This formulation of RPA follows the $\rho_{c}=0, \rho_{s} \neq 0$ case in ref. 29. Here the choice of $\phi_{s}$ is equivalent to an $\mathrm{NaCl}$ concentration of approximately $200 \mathrm{mM}$ such that the resulting electrostatic screening at $300 \mathrm{~K}$ is approximately equal to that entailed by the temperature-independent screening length $r_{\mathrm{S}}=10 \AA$ introduced in Models and Methods. (iii) RPA for a Coulomb potential with a short-range cutoff and temperature-independent screening, viz.,

$$
U_{i j}^{(\mathrm{S})}(r)=\frac{l_{B} \sigma_{i} \sigma_{j}}{r} e^{-r / r_{\mathrm{S}}}\left(1-e^{-r / a}\right)
$$

where $r$ is the spatial distance between charges $\sigma_{i}$ and $\sigma_{j}$. The Fourier transform $(k$-space expression) of $U_{i j}^{(\mathrm{S})}$ is given by

$$
\left(\hat{U}_{k}^{(\mathrm{S})}\right)_{i j}=l_{B} \sigma_{i} \sigma_{j}\left(\frac{4 \pi}{k^{2}+r_{\mathrm{S}}^{-2}}-\frac{4 \pi}{k^{2}+\left(1 / r_{\mathrm{S}}+1 / a\right)^{2}}\right)=\frac{4 \pi l_{B} \sigma_{i} \sigma_{j}\left(1+2 a / r_{\mathrm{S}}\right)}{\left(k^{2}+r_{\mathrm{S}}^{-2}\right)\left[(k a)^{2}+\left(a / r_{\mathrm{S}}+1\right)^{2}\right]} .
$$

The RPA-predicted free energy and phase behaviors for this model are readily obtained by replacing the $i, j$ elements of the matrix $\hat{U}_{k}$ in equation 35 of ref. 29 by the above expression for $\left(\hat{U}_{k}^{(\mathrm{S})}\right)_{i j}$ in Eq. 3. The coexistence phase boundaries predicted by these three RPA formulations for sequences sv1 and sv15 are shown in Fig. 10. The predominant trend predicted by all three formulations that $T_{\mathrm{cr}}$ is higher for sv15 than for sv1 are qualitatively consistent with our simulations but there are significant quantitative mismatches between theory and simulation. Our simulated results in Fig. 8 indicate that the ratio of the two polyampholytes' critical temperatures $T_{\mathrm{cr}}(\mathrm{sv} 15) / T_{\mathrm{cr}}(\mathrm{sv} 1) \approx 1.9$, a value that would not be affected by any potential temperature rescaling we entertained above. In contrast, the corresponding $T_{\mathrm{cr}}$ (sv15)/ $T_{\mathrm{cr}}$ (sv1) ratios predicted by the analytical theories are much larger: (i) 14.3 for salt-free RPA, (ii) 14.4 for RPA with monovalent salt, and (iii) 9.6 for RPA with temperature-independent screening. Another obvious simulation-theory discrepancy in Fig. 10 is that the theory-predicted critical volume fractions $\phi_{\mathrm{cr}}\left(\phi_{\mathrm{cr}}\right.$ 's are the $\phi$ values at $T_{\text {cr }}$ 's) are significantly lower than those estimated by our explicit-chain simulations.

As a control, we check whether adding an overall FH term in the RPA formulation by introducing a temperature-independent $\chi$ parameter in equation 10 of ref. 26 would result in a smaller mismatch with lattice simulation. One possible rationale for adding a FH term is to account for excluded volume effect that may not have been fully taken care of by RPA. We find that adding a repulsive FH term (which might be identified with a stronger excluded volume effect) leads to an even larger mismatch with the simulated $T_{\text {cr }}(\mathrm{sv} 15) / T_{\mathrm{cr}}$ (sv1) of approximately 1.9, whereas adding an attractive FH term (which might be caused by an overall background Lennard-Jones-like attraction among the monomers) leads to a smaller mismatch. But the shifts in the $T_{\mathrm{cr}}(\mathrm{sv} 15) / T_{\mathrm{cr}}(\mathrm{sv} 1)$ are small unless $|\chi|$ is very large. For 


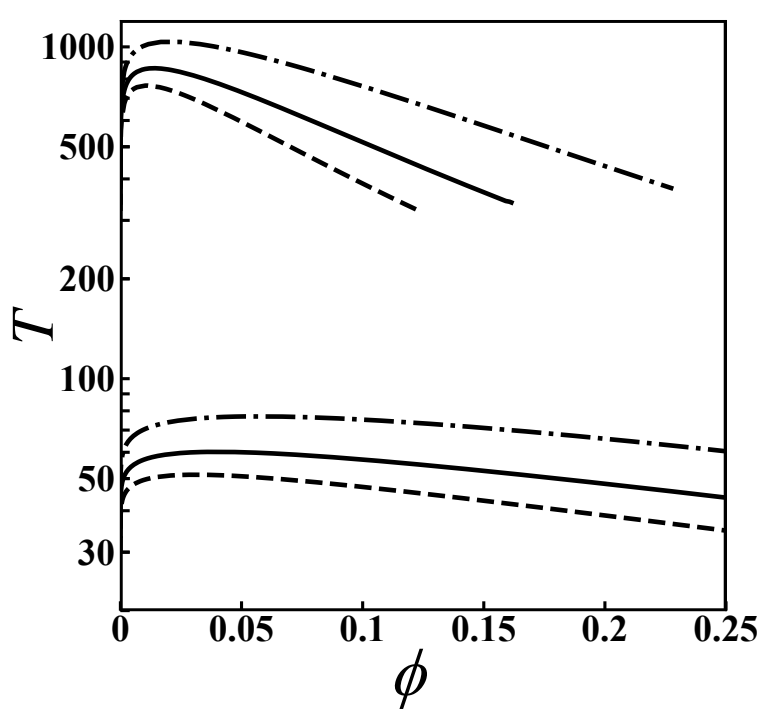

FIG. 11: RPA+FH theories. Phase boundaries for sequence sv1 (lower curves) and sequence sv15 (upper curves) in salt-free RPA (solid black curves), RPA $+\mathrm{FH}$ with $\chi=-0.5$ (dashed curves), and RPA + FH with $\chi=0.5$ (dashed-dotted curves).

instance, whereas $T_{\mathrm{cr}}(\mathrm{sv} 15) / T_{\mathrm{cr}}$ (sv1) for the original salt-free RPA is 14.3, the corresponding ratio is 14.9 with a repulsive $\chi=-0.5$, and 13.4 with an attractive $\chi=0.5$ (Fig. 11).

To better understand the simulation-theory mismatches for sv1 and sv15, it is instructive to place them in a broader perspective by extending the comparison to encompass previous explicit-chain lattice-simulations results on polyampholyte sequences. Literature reports on simulation studies of polyampholyte phase separations are quite limited, but we found the study by Cheong and Panagiotopoulos on a series of relatively short polyampholytes ${ }^{86}$ particularly useful for the present analysis. These authors constructed bond fluctuation models on simple cubic lattices for fully charged $N=2,4,8$, and 16 polyampholytes (all with zero net charge) to simulate their phase behaviors under the full Coulomb potential (no screening). Here we compare their simulation results for the four $N=8$ and three $N=16$ sequences they considered against the salt-free RPA results we obtain for the same sequences (Table 1). We contrast the simulation-theory mismatches for their sequences with those for our two $N=50$ sequences sv1 and sv15 by using the reduced temperature

$$
T^{*} \equiv \frac{a}{l_{B}}=\left(\frac{4 \pi \epsilon_{0} \epsilon_{\mathrm{r}} a}{e^{2}}\right) T
$$

to compare the critical temperatures of these models on the same footing. We also examine the degree to which the phase behaviors of these polyampholytes are correlated with the charge pattern parameters $\kappa$ (ref. 31) and SCD (ref. 32). 
Table 1. RPA-predicted critical parameters for the $N=8$ and 16 sequences in ref. 86

\begin{tabular}{|c|c|c|c|c|c|}
\hline Sequence $(\text { ref. } 86)^{a}$ & Charge pattern ${ }^{b}$ & $T_{\mathrm{cr}}^{*}$ & $\phi_{\mathrm{cr}}$ & SCD & $\kappa$ \\
\hline $\mathrm{P}_{4} \mathrm{~N}_{4}$ & KKKKEEEE & 0.339 & 0.0354 & -2.020 & 1.0000 \\
\hline $\mathrm{P}_{2} \mathrm{~N}_{2} \mathrm{P}_{2} \mathrm{~N}_{2}$ & KKEEKKEE & 0.125 & 0.0502 & -0.816 & 0.1331 \\
\hline $\mathrm{PN}_{3} \mathrm{PNP}_{2}$ & KEEEKEKK & 0.135 & 0.0481 & -0.849 & 0.5666 \\
\hline $\mathrm{P}_{2} \mathrm{~N}_{3} \mathrm{PNP}$ & KKEEEKEK & 0.117 & 0.0551 & -0.723 & 0.5666 \\
\hline $\mathrm{P}_{8} \mathrm{~N}_{8}$ & KKKKKKKKEEEEEEEE & 1.215 & 0.0242 & -5.240 & 1.0000 \\
\hline $\mathrm{P}_{4} \mathrm{~N}_{4} \mathrm{P}_{4} \mathrm{~N}_{4}$ & KKKKEEEEKKKKEEEE & 0.409 & 0.0326 & -1.802 & 0.0586 \\
\hline $\mathrm{PN}_{2} \mathrm{P}_{3} \mathrm{NPN}_{3} \mathrm{P}_{2} \mathrm{NPN}$ & KEEKKKEKEEEKKEKE & 0.143 & 0.0480 & -0.679 & 0.0233 \\
\hline
\end{tabular}

$a$ "P" and "N" denote, respectively, a positively and a negatively charged monomer.

${ }^{b}$ Same sequence in the notation of refs. $30-32$.

The results of our analysis are summarized in Fig. 12. The explicit-chain simulated $T_{\mathrm{cr}}^{*}$ and $\phi_{\text {cr }}$ values for the four $N=8$ and three $N=16$ sequences that are included in Fig. 12ac for comparison are taken from Table 1 of ref. 86. Fig. 12a shows that the RPA-predicted reduced critical temperatures ( $T_{\mathrm{cr}}^{*}$ 's) of polyampholytes of a given chain length $N$ correlate very well with the polyampholyte sequences' $\mathrm{SCD}$ values, as we have first reported for a set of thirty $N=50$ sequences ${ }^{30}$, the fitted linear $T_{\mathrm{cr}}^{*}-\mathrm{SCD}$ relation of which is reproduced here. Fig. 12a shows further that an approximate proportionality relationship $T_{\mathrm{cr}}^{*} \propto-\mathrm{SCD}$ holds for RPA-predicted $T_{\mathrm{cr}}^{*}$ values for a given $N$, with a proportionality constant that increases with $N$ : The slope of the fitted $T_{\mathrm{cr}}^{*}$ vs. $-\mathrm{SCD}$ line for $N=8$ is 0.165 (blue dashed line), that for $N=16$ is 0.231 (green dashed line), that for $N=50$ is 0.314 (black dotted line), that for $N=320$ is 0.384 (black dashed-dotted line). We also examine the twenty two RPA-predicted $T_{\text {cr }}^{*}$ values for the $N=120,240$, and 320 sequences that we considered previously ${ }^{29}$ as a whole (Fig. 12d) and find that their slopes are very similar $(0.379$ for $N=120,0.394$ for $N=240$ ), suggesting that the slope may limit to $\approx 0.40$ as $N \rightarrow \infty$.

Fig. 12a indicates that the explicit-chain simulated $T_{\text {cr }}^{*}$ 's for the $N=8$ and $N=16$ polyampholytes also correlate quite well with $\mathrm{SCD}$, but the increase of their $T_{\mathrm{cr}}^{*}$ with increasing value of $-\mathrm{SCD}$ is much more gradual than that predicted by RPA. For the case of the three $N=16$ sequences simulated by Cheong and Panagiotopoulos ${ }^{86}$, it is quite likely that the general approximate relationship is not linear but instead possesses a small but appreciable downward concavity. The degree of this simulation-theory mismatch in the slope $d \log \left(T_{\text {cr }}^{*}\right) / d(-\mathrm{SCD})$ appears to increase with chain length: According to Table 1 , the ratio between the highest and lowest RPA-predicted $T_{\text {cr }}^{*}$ 's is $0.339 / 0.117=2.90$ for $N=8$ and $1.215 / 0.143=8.50$ for $N=16$. The corresponding ratios from the explicit-chain simulation data of ref. 86 are, respectively, $0.361 / 0.240=1.50$ and $0.876 / 0.278=3.15$. This means that RPA overestimates the $T_{\text {cr }}^{*}$ ratio by a factor of $2.9 / 1.5=1.93$ for $N=8$ and 

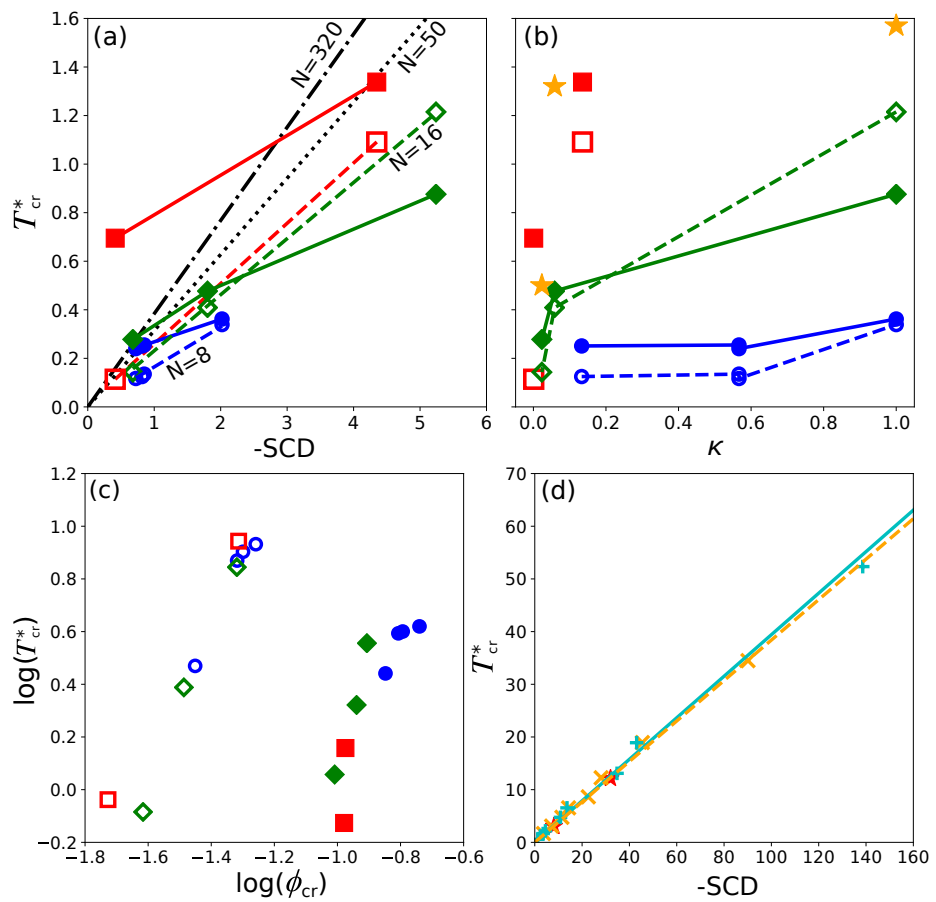

FIG. 12: Correlation of simulated and theory-predicted polyampholyte phase behaviors with charge pattern parameters. (a) Relationships between reduced critical temperature $T_{\mathrm{cr}}^{*}$ and the SCD parameter of Sawle and Ghosh ${ }^{32}$. Filled and open symbols represent, respectively, explicitchain simulation data and predictions by RPA theory. Red squares: Simulation results for sequences sv1 and sv15 from the present study and prediction by the RPA theory for Coulomb potential with temperature-independent screening (Eq.3. dashed-dotted curves in Fig. 10. Green diamonds: Simulated results and predictions by salt-free RPA theory for three different $N=16$ sequences in ref. 86. Blue circles: Simulated results and predictions by salt-free RPA theory for four different $N=8$ sequences in ref. 86. The simulation results for the $N=8$ and $N=16$ sequences are from Cheong and Panagiotopoulos ${ }^{86}$. These symbols carry the same meanings in (b) and (c). The simulated $T_{\mathrm{cr}}^{*}$ values for sequences sv1 and sv15 (filled red squares) are estimated from the empirically fitted phase boundaries in Fig. 8. Solid line segments drawn through the simulated data points and the dashed line through the two open red squares are guides for the eye. Other dashed lines are least-squares fits to the RPA data points depicted in the same color. Included for comparison are the fitted $T_{\mathrm{cr}}^{*}$ (for salt-free RPA) vs. -SCD relationship for the thirty $N=50$ sequences in ref. 30 (dotted line) and the eight $N=320$ sequences in ref. 29 (dashed-dotted line). (b) Dependence of $T_{\text {cr }}^{*}$ on the $\kappa$ parameter of Das and Pappu ${ }^{31}$. The three golden stars represent theoretical predictions by Jiang et al $\frac{108}{10}$ for the three $N=16$ sequences in ref. 85, the simulation and RPA-predicted results of which are depicted by green diamonds here and in (a). Solid and dashed lines segments drawn through the simulated and RPA data points, respectively, are guides for the eye. (c) Relationship between critical temperature $T_{\mathrm{cr}}^{*}$ and critical polymer volume fraction $\phi_{\mathrm{cr}}\left(\log =\log _{10}\right)$. The simulated $\phi_{\mathrm{cr}}$ values for sequences sv1 and sv15 (filled red squares) are estimated from the empirically fitted phase boundaries in Fig. 8. (d) $T_{\mathrm{cr}}^{*}$ predicted by salt-free RPA vs. $-\mathrm{SCD}$ for the two $N=120$ (red stars), twelve $N=240$ (turquoise plus signs), and eight $N=320$ (orange crosses) sequences in ref. 29. Lines are least-squares fits to the data points depicted in the same color. 
$8.50 / 3.15=2.70$ for $N=16$. Viewed in this context, the corresponding overestimation of $T_{\mathrm{cr}}$ (sv15)/ $T_{\mathrm{cr}}$ (sv1) by RPA with temperature-independent screening (the one physically most similar to our lattice model) by a factor of $9.6 / 1.9=5.1$ relative to the simulated value for our two $N=50$ polyampholytes (see above) fits quite well into the trend gleaned from our observations for the $N=8$ and $N=16$ sequences in Fig. 12a. Nonetheless, future studies that compare the present results against those from other explicit-chain models will be needed to ascertain the degree to which the trend seen here is sensitive to the pecularities of cubic lattice models.

Compared to the good correlations between explicit-chain simulated $T_{\mathrm{cr}}^{*}$ and $-\mathrm{SCD}$ in Fig. 12a, the correlations between explicit-chain simulated $T_{\mathrm{cr}}^{*}$ and $\kappa$ in Fig. 12b are less definitive. Three of the $N=8$ sequences have similar $T_{\mathrm{cr}}^{*}$ 's but one sequence has a $\kappa$ value much lower than that of the other two (the latter have identical $\kappa$ values, see filled blue circles in Fig. 12b). In contrast, SCD is better at capturing the small variations of $T_{\text {cr }}^{*}$ among these three sequences (filled blue circles in Fig. 12a). For the three longer $N=16$ sequences, $\kappa$ captures the general trend of how $T_{\mathrm{cr}}^{*}$ depends on sequence in that $\kappa$ increases monotonically with the explicit-chain simulated $T_{\mathrm{cr}}^{*}$. However, the $T_{\mathrm{cr}}^{*}-\kappa$ relationship (solid green lines in Fig. 12b) deviates much more from linearity than the corresponding $T_{\mathrm{cr}}^{*}-(-\mathrm{SCD})$ relationship (solid green lines in Fig. 12a), so much so that two of the $N=16$ sequences with appreciably different $T_{\mathrm{cr}}^{*}$ 's are seen to have very similarly low $\kappa$ values. The $\kappa$ parameter is an extremely useful intuitive measure of the blockiness of charge distribution along chain sequences ${ }^{31 \mid 106}$ (see, e.g., ref. 107 for a recent application). Because $\kappa$ relies on averaging charges over windows of 5-6 residues, it is not entirely surprising that the effectiveness of this parameter would diminish for short sequences with lengths not much longer than the window for charge averaging. A clear strength of SCD (Eq. 1) is its ability to account for patterning features that encompass charges that are far apart along the chain sequence. SCD emerges from a field-theoretic variational approach to account for sequence-dependent single-polyampholyte conformational dimensions by renormalized Kuhn lengths ${ }^{32}$. In this theory, the average end-to-end distance is a function of SCD; but the corresponding expression for average radius of gyration involves additional charge-pattern terms (cf. equations 6 and 13 of ref. 32). Whereas the extremely good correlations between SCD and RPApredicted $T_{\mathrm{cr}}^{*}$ might be partly attributed to the underlying Gaussian chain model shared by both the variational and RPA theories (though the detailed mathematical relationship remains to be explored), it is quite remarkable that the simple SCD parameters are also well correlated with explicit-chain simulated single-polyampholyte radii of gyration 30131 as well as $T_{\mathrm{cr}}^{*}$ 's for multiple-chain phase separations (Fig. 12a). It would be very instructive to elucidate what nuanced effects beyond an intuitive characterization of charge blockiness are captured by the SCD parameter.

For completeness, we also include the $T_{\mathrm{cr}}^{*}$ 's for the three $N=16$ sequences predicted by 
Jiang et al.'s charged hard-sphere chain model theory ${ }^{108}$ which makes use of an analytical hard-sphere equation of state ${ }^{109}$ (golden stars in Fig. 12b). These theoretically predicted $T_{\mathrm{cr}}^{*}$ 's correlate quite well with their explicit-chain simulated counterparts (filled green diamonds in Fig. 12b), suggesting that this theory can be a useful general approach to study sequence-dependent phase behaviors.

Fig. 12c compares the explicit-chain simulated and RPA-predicted critical volume fractions $\left(\phi_{\text {cr }}\right)$. RPA significantly underestimates $\phi_{\text {cr }}$ for all nine polyampholytes sequences considered. The $\phi_{\text {cr }}$ values estimated from explicit-chain simulations of the two $N=50$ sequences studied here (filled red squares) are comparable to the explicit-chain simulated $\phi_{\text {cr }}$ values for the $N=8$ and $N=16$ sequences (filled green and blue symbols). As has been commented upon ${ }^{92}$, field-theoretic approaches to polyelectrolyte and polyampholyte phase separations tend to significantly underestimate $\phi_{\text {cr }}$ because the analytical treatments of density fluctuation is not sufficiently accurate. A case in point is that RPA predicts extremely low $\phi_{\text {cr }}$ 's for polyelectrolytes with neutralizing counterions ${ }^{2728}$. Even with an improved analytical treatment ${ }^{1100}$, the predicted $\phi_{\mathrm{cr}}$ 's are still lower than that obtained by explicit-chain simulations ${ }^{85}$. We deem it likely that the underlying Gaussin chain model of RPA leads to underestimations of interchain attraction and thus a general underestimation of $\phi_{\mathrm{cr}}$. By the same token, the underlying Gaussian chain model does not fully account for the possibilities that strong interchain attractions are achievable by spatially pairing up low- $|\mathrm{SCD}|$ sequences in certain specific configurations. This limitation probably contributes to an underestimation of the propensities of low- $|\mathrm{SCD}|$ sequences to phase separate and thus a much sharper dependence of $T_{\mathrm{cr}}^{*}$ on $-\mathrm{SCD}$ than that revealed by explicit-chain simulations (Figs. 10 and 12a). This idea remains to be tested. It deserves further investigations that consider a set of sequences more extensive than that studied here. All in all, analytical theories are extremely useful conceptual tools and are valuable for predicting and rationalizing trends in sequence-dependent phase separations that are consistent with explicit-chain simulations (Fig. 12) and experiments ${ }^{26}$. Nonetheless, addressing the aforementioned limitations would be necessary to improve their quantitative accuracy.

\section{Conclusions}

To recapitulate, we have presented explicit-chain simulation data for $n=300$ copies of fully charge polyampholytes with $N=50$ monomers configured on simple cubic lattices for an extensive set of temperatures and overall polyampholyte concentrations. A comparison of the results for two specific polyampholyte sequences with significantly different charge patterns indicates that the sequence with a more blocky charge pattern has a significantly higher tendency to phase separate. While this trend is anticipated and is consistent with 
predictions by RPA theory, the variation of phase-separating tendency with charge pattern is milder in the present lattice model than that predicted by RPA. Similar qualitative agreements and quantitative mismatches between explicit-chain simulation and RPA are identified for several shorter polyampholyte sequences that have been simulated previously for their phase properties. Taken together, these findings lend credence to the utility of RPA as an important tool for conceptual development and qualitative predictions. At the same time, they underscore that caution should be exercised in quantitative interpretation of predictions from RPA and other analytical theories. Our analysis suggests that sequence charge decoration (SCD) is an effective parameter for capturing the phase separating tendency of polyampholytes with zero net charge, but the underlying physical reasons for its success remain to be better elucidated. We have also compared the phase behaviors of an all-hydrophobic sequence and a largely hydrophobic sequence with sparsely embedded negative charges as a toy model for exploring effects of phosphorylations on IDP phase separations and to provide a simple rationalization for pertinent experimental observations. Promising future extensions of the present effort include incorporation of structurally and energetically more detailed representations of the interactions as well as construction of continuum (off-lattice) models for sequence-dependent phase behaviors. Adaptation of

a recently developed simulation technique for Lennard-Jones homopolymers ${ }^{111}$ should be particularly useful in this regard.

\section{Acknowledgments}

We thank Lewis Kay and Heinrich Krobath for helpful discussions, and members and trainees of the National Science Foundation (NSF)-funded Protein Folding and Dynamics Research Coordination Network for insightful inputs during the Network's June-2017 Annual Meeting at UC Berkeley where an earlier version of this work was presented by S.D. (NSF grant MCB 1516959). This work was supported by Canadian Cancer Society Research Institute grant no. 703477, Canadian Institutes of Health Research grant MOP-84281, and computational resources provided by SciNet of Compute/Calcul Canada. 


\section{References}

1 Maynard Smith, J. The concept of information in biology. Phil. Sci. 2000, 67, 177-194.

2 Uversky, V. N.; Oldfield, C. J.; Dunker, A. K. Intrinsically disordered proteins in human diseases: Introducing the $\mathrm{D}^{2}$ concept. Annu. Rev. Biophys. 2008, 37, 215-246.

3 Tompa, P. Intrinsically unstructured proteins: a 10-year recap. Trends Biochem. Sci. 2012, 37, 509-516.

4 Wright, P. E.; Dyson, H. J. Intrinsically disordered proteins in cellular signalling and regulation. Nat. Rev. Mol. Cell Biol. 2015, 16, 18-29.

5 Chen, J. Towards the physical basis of how intrinsically disorder mediates protein function. Arch. Biochem. Biophys. 2012, 524, 123-131.

6 Chen, T.; Song, J.; Chan, H. S. Theoretical perspectives on nonnative interactions and intrinsic disorder in protein folding and binding. Curr. Opin. Struct. Biol. 2015, 30, 32-42.

7 Salonen, L. M.; Ellermann, M.; Diederich, F. Aromatic rings in chemical and biological recognition: Energetics and structures. Angew. Chem. Int. Ed. 2011, 50, 4808-4842.

8 Brangwynne, C. P.; Mitchison, T. J.; Hyman, A. A. Active liquid-like behavior of nucleoli determines their size and shape in Xenopus laevis oocytes. Proc. Natl. Acad. Sci. U.S.A. 2011, 108, 4334-4339.

9 Hyman, A. A.; Weber, C. A.; Jülicher, F. Liquid-liquid phase separation in biology. Annu. Rev. Cell Dev. Biol. 2014 30, 39-58.

10 Toretsky, J. A.; Wright, P. E. Assemblages: Functional units formed by cellular phase separation. J. Cell Biol. 2014, 206, 579-588.

$11 \mathrm{Wu}, \mathrm{H} . ;$ Fuxreiter, M. The structure and dynamics of higher-order assemblies: Amyloids, signalosomes, and granules. Cell 2016 165, 1055-1066.

12 Chong, P. A.; Forman-Kay, J. D. Liquid-liquid phase separation in cellular signaling systems. Curr Opin Struct Biol 2016, 41, 180-186.

13 Mitrea, D. M.; Kriwacki, R. W. Phase separation in biology; functional organization of a higher order. Cell Commun. Signal. 2016, 14, 1.

14 Shin, Y.; Brangwynne, C. P. Liquid phase condensation in cell physiology and disease. Science 2017, 357, eaaf4382.

15 Nott, T. J.; Petsalaki, E.; Farber, P.; Jervis, D.; Fussner, E.; Plochowietz, A.; Craggs, T. D.; Bazett-Jones, D. P.; Pawson, T.; Forman-Kay, J. D.; et al. Phase transition of a disordered nuage protein generates environmentally responsive membraneless organelles. Mol. Cell 2015, 57, 936-947. 
16 Feric, M.; Vaidya, N.; Harmon, T. S.; Mitrea, D. M.; Zhu, L.; Richardson, T. M.; Kriwacki, R. W.; Pappu, R. V.; Brangwynne, C. P. Coexisting liquid phases underlie nucleolar subcompartments. Cell 2016 165, 1686-1697.

17 Riback, J. A.; Katanski, C. D.; Kear-Scott, J. L.; Pilipenko, E. V.; Rojek, A. E.; Sosnick T. R.; Drummond, D. A. Stress-triggered phase separation is an adaptive, evolutionarily tuned response. Cell 2017, 168, 1028-1040.

18 Brady, J. P.; Farber, P. J.; Sekhar, A.; Lin, Y.-H.; Huang, R.; Bah, A.; Nott, T. J.; Chan, H. S.; Baldwin, A. J.; Forman-Kay, J, D.; et al. Structural and hydrodynamic properties of an intrinsically disordered region of a germ-cell specific protein on phase separation. Proc. Natl. Acad. Sci. USA 2017, 114, E8194-E8203.

19 Kato, M.; Han, T. W.; Xie, S.; Shi, K.; Du, X.; Wu, L. C.; Mirzaei, H.; Goldsmith, E. J.; Longgood, J.; Pei, J.; et al. Cell-free formation of RNA granules: Low complexity sequence domains form dynamic fibers within hydrogels. Cell 2012, 149, 753-767.

20 Lin, Y.; Protter, D. S. W.; Rosen, M. K.; Parker, R. Formation and maturation of phaseseparated droplets by RNA-binding proteins. Mol. Cell 2015, 60, 208-219.

21 Jain, S.; Wheeler, J. R.; Walters, R. W.; Agrawal, A.; Barsic, A.; Parker, R. ATPasemodulated stress granules contain a diverse proteome and substructures. Cell 2016, 164, 487-498.

22 Muiznieks, L. D.; Cirulis, J. T.; van der Horst, A.; Reinhardt, D. P.; Wuite, G. J. L.; Pomès, R.; Keeley, F. W. Modulated growth, stability and interactions of liquid-like coacervate assemblies of elastin. Matrix Biol. 2014, 36, 39-50.

23 Molliex, A.; Temirov, J.; Lee, J.; Coughlin, M.; Kanagaraj, A. P.; Kim, H. J.; Mittag, T.; Taylor, J. P. Phase separation by low complexity domains promotes stress granule assembly and drives pathological fibrillization. Cell 2015, 163, 123-133.

24 Quiroz, F. G.; Chilkoti, A. Sequence heuristics to encode phase behaviour in intrinsically disordered protein polymers. Nat. Mater. 2015, 14, 1164-1171.

25 Pak, C. W.; Kosno, M.; Holehouse, A. S.; Padrick, S. B.; Mittal, A.; Ali, R.; Yunus, A. A.; Liu, D. R.; Pappu, R. V.; Rosen M. K. Sequence determinants of intracellular phase separation by complex coacervation of a disordered protein. Mol. Cell 2016, 63, 72-85.

26 Lin, Y.-H.; Forman-Kay, J. D.; Chan, H. S. Sequence-specific polyampholyte phase separation in membraneless organelles. Phys. Rev. Lett. 2016, 117, 178101.

27 Mahdi, K. A.; Olvera de la Cruz, M. Phase diagrams of salt-free polyelectrolyte semidilute solutions. Macromolecules 2000, 33, 7649-7654.

28 Ermoshkin, A. V.; Olvera de la Cruz, M. A modified random phase approximation of polyelectrolyte solutions. Macromolecules 2003, 36, 7824-7832.

29 Lin, Y.-H.; Song, J.; Forman-Kay, J. D.; Chan, H. S. Random-phase-approximation theory for sequence-dependent, biologically functional liquid-liquid phase separation of intrinsically 
disordered proteins. J. Mol. Liq. 2017, 228, 176-193.

30 Lin, Y.-H.; Chan, H. S. Phase separation and single-chain compactness of charged disordered proteins are strongly correlated. Biophys, J. 2017, 112, 2043-2046.

31 Das, R. K.; Pappu, R. V. Conformations of intrinsically disordered proteins are influenced by linear sequence distribution of oppositely charged residues. Proc. Natl. Acad. Sci. U.S.A. 2013, 110, 13392-13397.

32 Sawle, L.; Ghosh, K. A theoretical method to compute sequence dependent configurational properties in charged polymers and proteins. J. Chem. Phys. 2015, 143, 085101.

33 Sawle, L.; Huihui, J.; Ghosh, K. All-atom simulations reveal protein charge decoration in the folded and unfolded ensemble is key in thermophilic adaptation. J. Chem. Theor. Comput. 2017, 13, 5065-5075.

34 Firman, T.; Ghosh, K. Sequence charge decoration dictates coil-globule transition in intrinsically disordered proteins. J. Chem. Phys. 2018, 148, 123305.

35 Lin, Y.-H.; Brady, J. P.; Forman-Kay, J. D.; Chan, H. S. Charge pattern matching as a 'fuzzy' mode of molecular recognition for the functional phase separations of intrinsically disordered proteins. New J. Phys. 2017, 19, 115003.

36 Chang, L.-W.; Lytle, T. K.; Radhakrishna, M.; Madinya, J. J.; Vélez, J.; Sing, C. E.; Perry, S. L. Sequence and entropy-based control of complex coacervates. Nat. Comm. 2017, 8, 1273.

37 Das, R. K.; Ruff, K. M.; Pappu, R. V. Relating sequence encoded information to form and function of intrinsically disordered proteins. Curr. Opin. Struct. Biol. 2015, 32, 102-112.

Best, R. B. Computational and theoretical advances in studies of intrinsically disordered proteins. Curr. Opin. Struct. Biol. 2017, 42,147-154.

39 Levine, Z. A.; Shea, J.-E. Simulations of disordered proteins and systems with conformational heterogeneity. Curr. Opin. Struct. Biol. 2017, 43, 95-103.

40 Ruff, K. M.; Harmon, T. S.; Pappu, R. V. CAMELOT: A machine learning approach for coarse-grained simulations of aggregation of block-copolymeric protein sequences. J. Chem. Phys. 2015, 143, 243123.

41 Orr, W. J. C. Statistical treatment of polymer solutions at infinite dilution. Trans. Faraday Soc. 1947, 43, 12-27.

42 Domb, C. Self avoiding walks on lattices. Adv. Chem. Phys. 1969, 15, 229-259.

43 de Gennes, P.-G. Scaling Concepts in Polymer Physics; Cornell University Press, Ithaca, U.S.A.; 1979; pp 39-43.

44 Larson, R. G.; Scriven, L. E.; Davis, H. T. Monte Carlo simulation of model amphiphile-oilwater systems. J. Chem. Phys. 1985, 83, 2411-2420.

45 Sumners, D. W.; Whittington, S. G. Knots in self-avoiding walks. J. Phys. A.-Math. Gen. 1988, 21, 1689-1694.

46 Chen, S.-J.; Dill, K. A. Statistical thermodynamics of double-stranded polymer molecules. $J$. 
Chem. Phys. 1995, 103, 5802-5813.

47 Cao, S.; Chen, S.-J. Predicting RNA pseudoknot folding thermodynamics. Nucl. Acids Res. 2006, 34, 2634-2652.

48 Liu, Z.; Mann, J. K.; Zechiedrich, E. L.; Chan, H. S. Topological information embodied in local juxtaposition geometry provides a statistical mechanical basis for unknotting by type-2 DNA topoisomerases. J. Mol. Biol. 2006, 361, 268-285.

49 Taketomi, H.; Ueda, Y.; Gō, N. Studies on protein folding, unfolding and fluctuations by computer simulation. I. The effect of specific amino acid sequence represented by specific inter-unit interactions. Int. J. Pept. Protein Res. 1975, 7, 445-459.

50 Taketomi, H.; Kanô, F.; Gō, N. The effect of amino acid substitution on protein-folding and -unfolding transition studied by computer simulation. Biopolymers 1988, 27, 527-559.

51 Gō, N.; Taketomi, H. Respective roles of short- and long-range interactions in protein folding. Proc. Natl. Acad. Sci. U.S.A. 1978, 75, 559-563.

52 Chan, H. S. Protein folding: Matching speed and locality. Nature 1998 392, 761-763.

53 Gō, N. Theoretical studies of protein folding. Annu. Rev. Biophys. Bioeng. 1983, 12, 183-210.

54 Go, N. The Consistency Principle Revisited. In Old and New Views of Protein Folding; Kuwajima, K., Arai, M., Eds.; Elsevier, Amsterdam, The Netherlands, 1999; pp 97-105.

55 Bryngelson, J. D.; Wolynes, P. G. Spin glasses and the statistical mechanics of protein folding. Proc. Natl. Acad. Sci. U.S.A. 1987, 84, 7524-7528.

56 Chan, H. S.; Zhang, Z.; Wallin, S.; Liu, Z. Cooperativity, local-nonlocal coupling, and nonnative interactions: Principles of protein folding from coarse-grained models. Annu. Rev. Phys. Chem. 2011, 62, 301-326.

57 Lau, K. F.; Dill, K. A. A lattice statistical mechanics model of the conformational and sequence spaces of proteins. Macromolecules 1989, 22, 3986-3997.

58 Dill, K. A. Theory for the folding and stability of globular proteins. Biochemistry 1985, 24, 1501-1509.

59 Lau, K. F.; Dill, K. A. Theory for protein mutability and biogenesis. Proc. Natl. Acad. Sci. U.S.A. 1990, 87, 638-642.

60 O'Toole, E. M.; Panagiotopoulos, A. Z. Monte Carlo simulation of folding transitions of simple model proteins using a chain growth algorithm. J. Chem. Phys. 1992, 97, 8644-8652.

61 Lipman, D. J.; Wilbur, W. J. Modelling neutral and selective evolution of protein folding. Proc. R. Soc. Lond. B 1991, 245, 7-11.

62 Chan, H. S.; Bornberg-Bauer, E. Perspectives on protein evolution from simple exact models. Appl. Bioinform. 2002, 1, 121-144.

63 Chan, H. S.; Dill, K. A. Intrachain loops in polymers: Effects of excluded volume. J. Chem. Phys. 1989, 90, 492-509.

64 Chan, H. S.; Dill, K. A. The effects of internal constraints on the configurations of chain 
molecules. J. Chem. Phys. 1990, 92, 3118-3135; Erratum: J. Chem. Phys. 1997, 107, 10353.

Chan, H. S.; Dill, K. A. Compact polymers. Macromolecules 1989, 22, 4559-4573.

Camacho, C. J.; Thirumalai, D. Minimum energy compact structures of random sequences of heteropolymers. Phys. Rev. Lett. 1993, 71, 2505-2508.

67 Shakhnovich, E.; Farztdinov, G.; Gutin, A. M.; Karplus, M. Protein folding bottlenecks: A lattice Monte Carlo simulation. Phys. Rev. Lett. 1991, 67, 1665-1668.

68 Leopold, P. E.; Montal, M.; Onuchic, J. N. Protein folding funnels: A kinetic approach to the sequence-structure relationship. Proc. Natl. Acad. Sci. U.S.A. 1992, 89, 8721-8725.

69 Wolynes, P. G.; Onuchic, J. N.; Thirumalai, D. Navigating the folding routes. Science 1995, 267, 1619-1620.

70 Chan, H. S.; Shimizu, S.; Kaya, H. Cooperativity principles in protein folding. Methods Enzymol. 2004, 380, 350-379.

71 Li, H.; Helling, R.; Tang, C.; Wingreen, N. Emergence of preferred structures in a simple model of protein folding. Science 1996, 273, 666-669.

72 Shakhnovich, E. I. Proteins with selected sequences fold into unique native conformation. Phys. Rev. Lett. 1994, 72, 3907-3910.

73 Covell, D. G.; Jernigan, R. L. Conformations of folded proteins in restricted spaces. Biochemistry 1990, 29, 3287-3294.

74 Kolinski, A.; Skolnick, J.; Yaris, R. The collapse transition of semiflexible polymers. A Monte Carlo simulation of a model system. J. Chem. Phys. 1986, 85, 3585-3597.

75 Hinds, D. A.; Levitt, M. A lattice model for protein structure prediction at low resolution. Proc. Natl. Acad. Sci. U.S.A. 1992, 89, 2536-2540.

76 Skolnick, J.; Kolinski, A. Simulations of the folding of a globular protein. Science 1990, 250, 1121-1125.

77 Dill, K. A.; Bromberg, S.; Yue, K.; Fiebig, K. M.; Yee, D. P.; Thomas, P. D.; Chan, H. S. Principles of protein folding - A perspective from simple exact models. Protein Sci. 1995, 4, 561-602.

78 Bryngelson, J. D.; Onuchic, J. N.; Socci, N. D.; Wolynes, P. G. Funnels, pathways, and the energy landscape of protein folding: A synthesis. Proteins 1995, 21, 167-195.

79 Chan, H. S.; Kaya, H.; Shimizu, S. Computational Methods for Protein Folding: Scaling a Hierarchy of Complexities. In Current Topics in Computational Molecular Biology; Jiang, T., Xu Y., Zhang, M. Q., Eds.; The MIT Press: Cambridge, Massachusetts, U.S.A., 2002; Chapter 16, pp 403-447.

80 Guseva, E.; Zuckermann, R. N.; Dill, K. A. Foldamer hypothesis for the growth and sequence differentiation of prebiotic polymers. Proc. Natl. Acad. Sci. U.S.A. 2017, 114, E7460-E7468.

81 Moreno-Hernández, S.; Levitt, M. Comparative modeling and protein-like features of hydrophobic-polar models on a two-dimensional lattice. Proteins 2012, 80, 1683-1693. 
Sikosek, T.; Chan, H. S. Biophysics of protein evolution and evolutionary protein biophysics. J. R. Soc. Interface 2014, 11, 20140419,

83 Higgs, P. G.; Joanny, J. F. Theory of polyampholyte solutions. J. Chem. Phys. 1991 94, $1543-1554$.

84 Wittmer, J.; Johner, A.; Joanny, J. F. Random and alternating polyampholytes. EPL 1993, 24, 263-268.

85 Orkoulas, G.; Kumar, S. K.; Panagiotopoulos, A. Z. Monte Carlo study of Coulombic criticality in polyelectrolytes. Phys. Rev. Lett. 2003, 90, 048303.

86 Cheong, D. W.; Panagiotopoulos, A. Z. Phase behaviour of polyampholyte chains from grand canonical Monte Carlo simulations. Mol. Phys. 2005, 103, 3031-3044.

87 Panagiotopoulos, A. Z.; Wong, V.; Floriano, M. A. Phase equilibria of lattice polymers from histogram reweighting Monte Carlo simulations. Macromolecules 1998, 31, 912-918.

88 Carmesin, I.; Kremer, K. The bond fluctuation method: a new effective algorithm for the dynamics of polymers in all spatial dimensions. Macromolecules 1988, 21, 2819-2823.

89 Baschnagel, J.; Wittmer, J. P.; Meyer, H. Monte Carlo simulation of polymers: Coarsegrained models. In Computational Soft Matter: From Synthetic Polymers to Proteins; Attig, N.; Binder, K.; Grubmüller, H.; Kremer K.. Eds.; John von Neumann Institute for Computing, Jülich, Germany, 2004; pp 83-140.

90 White, A. D.; Voth, G. A. Efficient and minimal method to bias molecular simulations with experimental data. J. Chem. Theor. Comput. 2014, 10, 3023-3030.

91 Wang, Z.-G. Concentration fluctuation in binary polymer blends: $\chi$ parameter, spinodal and Ginzburg criterion. J. Chem. Phys. 2002, 117, 481-500.

92 Wang, R.; Wang, Z.-G. Theory of polymer chains in poor solvents: Single-chain structure, solution thermodynamics, and $\Theta$ point. Macromolecules 2014, 47, 4094-4102.

93 Chan, H. S.; Dill, K. A. Transition states and folding dynamics of proteins and heteropolymers. J. Chem. Phys. 1994, 100, 9238-9257.

94 Yue, K.; Fiebig, K. M.; Thomas, P. D.; Chan, H. S.; Shakhnovich, E. I.; Dill, K. A. A test of lattice protein folding algorithms. Proc. Natl. Acad. Sci. U.S.A. 1995, 92, 325-329.

95 Kaya H.; Chan, H. S. Origins of chevron rollovers in non-two-state protein folding kinetics. Phys. Rev. Lett. 2003, 90, 258104.

96 Rey, A.; Skolnick, J. Comparison of lattice Monte Carlo dynamics and Brownian dynamics folding pathways of $\alpha$-helical hairpins. Chem. Phys. 1991, 158, 199-219.

97 Socci, N. D.; Bialek, W. S.; Onuchic, J. N. Properties and origins of protein secondary structure. Phys. Rev. E 1994, 49, 3440-3443.

98 Hunt, N. G.; Gregoret, L. M.; Cohen, F. E. The origins of protein secondary structure: Effects of packing density and hydrogen bonding studied by a fast conformational search. J. Mol. Biol. 1994, 241, 312-326. 
Yee, D. P.; Chan, H. S.; Havel, T. F.; Dill, K. A. Does compactness induce secondary structure in proteins? A study of poly-alanine chains computed by distance geometry. J. Mol. Biol. 1994, 241, 557-573.

100 Dill, K. A.; Alonso, D. O. V.; Hutchinson, K. Thermal stabilities of globular proteins. Biochemistry 1989 28, 5439-5449.

101 Shimizu, S.; Chan, H. S. Configuration-dependent heat capacity of pairwise hydrophobic interactions. J. Am. Chem. Soc. 2001, 123, 2083-2084.

102 Borg, M.; Mittag, T.; Pawson, T.; Tyers, M.; Forman-Kay, J. D.; Chan, H. S. Polyelectrostatic interactions of disordered ligands suggest a physical basis for ultrasensitivity. Proc. Natl. Acad. Sci. U.S.A. 2007, 104, 9650-9655.

103 Csizmok, V.; Orlicky, S.; Cheng, J.; Song, J.; Bah, A.; Delgoshaie, N.; Lin, H.; Mittag, T.; Sicheri, F.; Chan, H. S.; et al. An allosteric conduit facilitates dynamic multisite substrate recognition by the $\mathrm{SCF}^{\mathrm{Cdc} 4}$ ubiquitin ligase. Nat. Comm. 2017, 8, 13943.

104 Kwon, I.; Kato, M.; Xiang, S.; Wu, L.; Theodoropoulos, P.; Mirzaei, H.; Han, T.; Xie, S.; Corden, J. L.; McKnight, S. L. Phosphorylation-regulated binding of RNA polymerase II to fibrous polymers of low-complexity domains. Cell 2013, 155, 1049-1060.

105 Monahan, Z.; Ryan, V. H.; Janke, A. M.; Burke, K. A.; Rhoads, S. N.; Zerze, G. H.; O’Meally, R.; Dignon, G. L.; Conicella, A. E.; Zheng, W.; et al. Phosphorylation of the FUS lowcomplexity domain disrupts phase separation, aggregation, and toxicity. EMBO J. 2017, 36, 2951-2967.

106 Holehouse, A. S.; Das, R. K.; Ahad, J. N.; Richardson, M. O. G.; Pappu, R. V. CIDER: Resources to analyze sequence-ensemble relationships of intrinsically disordered proteins. Biophys. J. 2017, 112, 16-21.

107 Sherry, K. P.; Das, R. K.; Pappu, R. V.; Barrick, D. Control of transcriptional activity by design of charge patterning in the intrinsically disordered RAM region of the Notch receptor. Proc. Natl. Acad. Sci. U.S.A. 2017, 114, E9243-E9252.

108 Jiang, J.; Feng, J.; Liu, H.; Hu, Y. Phase behavior of polyampholytes from charged hard-sphere chain model. J. Chem. Phys. 2006, 124, 144908.

109 Boublík, T. Hard-sphere equation of state. J. Chem. Phys. 1970, 53, 471-472.

110 Muthukumar, M. Phase diagram of polyelectrolyte solutions: Weak polymer effect. Macromolecules 2002, 35, 9142-9145.

111 Silmore, K. S.; Howard, M. P.; Panagiotopoulos, A. Z. Vapour-liquid phase equilibrium and surface tension of fully flexible Lennard-Jones chains. Mol. Phys. 2017, 115, 320-327. 

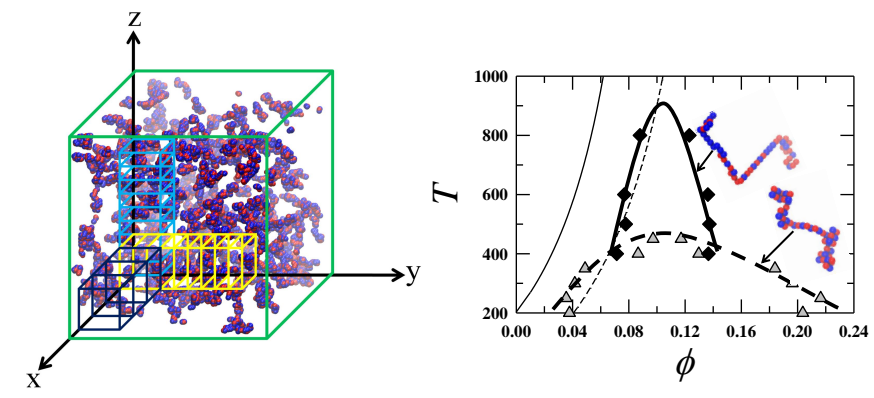

TOC Graphic 